\title{
Myelodysplastic syndromes are induced by histone methylation-altering ASXL1 mutations
}

Daichi Inoue, ${ }^{1}$ Jiro Kitaura, ${ }^{1}$ Katsuhiro Togami, ${ }^{1}$ Koutarou Nishimura, ${ }^{1}$ Yutaka Enomoto, ${ }^{1}$ Tomoyuki Uchida, ${ }^{1}$ Yuki Kagiyama, ${ }^{1}$ Kimihito Cojin Kawabata, ${ }^{1}$ Fumio Nakahara, ${ }^{1}$ Kumi Izawa, ${ }^{1}$ Toshihiko Oki, ${ }^{1,2}$ Akie Maehara, ${ }^{1}$ Masamichi Isobe, ${ }^{1}$ Akiho Tsuchiya, ${ }^{1}$ Yuka Harada, ${ }^{3}$ Hironori Harada, ${ }^{4}$ Takahiro Ochiya, ${ }^{5}$ Hiroyuki Aburatani, ${ }^{6}$ Hiroshi Kimura, ${ }^{7}$ Felicitas Thol, ${ }^{8}$ Michael Heuser, ${ }^{8}$ Ross L. Levine, ${ }^{9}$ Omar Abdel-Wahab, ${ }^{9}$ and Toshio Kitamura ${ }^{1,2}$

\author{
1Division of Cellular Therapy, Advanced Clinical Research Center, and 'Division of Stem Cell Signaling, Center for Stem Cell Biology and Regenerative Medicine, \\ Institute of Medical Science, University of Tokyo, Tokyo, Japan. ${ }^{3}$ Division of Radiation Information Registry and \\ ${ }^{4}$ Department of Hematology and Oncology, Research Institute for Radiation Biology and Medicine, Hiroshima University, Hiroshima, Japan. \\ ${ }^{5}$ Division of Molecular and Cellular Medicine, National Cancer Center Research Institute, Tokyo, Japan. \\ ${ }^{6}$ Genome Science Division, Research Center for Advanced Science and Technology, University of Tokyo, Tokyo, Japan. \\ ${ }^{7}$ Graduate School of Frontier Biosciences, Osaka University, Osaka, Japan. \\ ${ }^{8}$ Department of Hematology, Hemostasis, Oncology, and Stem Cell Transplantation, Hannover Medical School, Hannover, Germany. \\ ${ }^{9}$ Human Oncology and Pathogenesis Program and Leukemia Service, Department of Medicine, \\ Memorial Sloan-Kettering Cancer Center, New York, New York, USA.
}

\begin{abstract}
Recurrent mutations in the gene encoding additional sex combs-like 1 (ASXL1) are found in various hematologic malignancies and associated with poor prognosis. In particular, ASXL1 mutations are common in patients with hematologic malignancies associated with myelodysplasia, including myelodysplastic syndromes (MDSs), and chronic myelomonocytic leukemia. Although loss-of-function ASXL1 mutations promote myeloid transformation, a large subset of ASXL1 mutations is thought to result in stable truncation of ASXL1. Here we demonstrate that C-terminal-truncating Asxl1 mutations (ASXL1-MTs) inhibited myeloid differentiation and induced MDS-like disease in mice. ASXL1-MT mice displayed features of human-associated MDS, including multilineage myelodysplasia, pancytopenia, and occasional progression to overt leukemia. ASXL1-MT resulted in derepression of homeobox A9 (Hoxa9) and microRNA-125a (miR-125a) expression through inhibition of polycomb repressive complex 2-mediated (PRC2-mediated) methylation of histone H3K27. miR-125a reduced expression of C-type lectin domain family 5 , member a (Clec5a), which is involved in myeloid differentiation. In addition, HOXA9 expression was high in MDS patients with ASXL1-MT, while CLEC5A expression was generally low. Thus, ASXL1-MT-induced MDS-like disease in mice is associated with derepression of Hoxa9 and miR-125a and with Clec5a dysregulation. Our data provide evidence for an axis of MDS pathogenesis that implicates both ASXL1 mutations and miR-125a as therapeutic targets in MDS.
\end{abstract}

\section{Introduction}

Additional sex combs-like 1 (ASXL1) is 1 of 3 mammalian homologs of the Drosophila additional sex combs (Asx), and plays critical roles both in activation and suppression of Hox genes in axial patterning through regulating the polycomb group and trithorax group proteins (1-4). ASXL1 is mutated in patients with the entire spectrum of myeloid malignancies including $11 \%-21 \%$ of patients with myelodysplastic syndrome (MDS) (5-8), 10\%-15\% of patients with myeloproliferative neoplasms (MPNs), $5 \%-25 \%$ of patients with acute myeloid leukemia (AML) $(5,7)$, and $43 \%$ $58 \%$ of patients with chronic myelomonocytic leukemia (CMML) $(6,7,9,10)$. Additionally, ASXL1 mutations are associated with adverse survival in a variety of myeloid malignancies $(8,9)$.

Recently it was reported that ASXL1 binds members of the polycomb repressive complex 2 (PRC2), specifically EZH2, EED, and SUZ12, and that ASXL1 loss in myeloid hematopoietic cells profoundly inhibits trimethylation of histone $\mathrm{H} 3$-lysine 27 (H3K27me3), a hallmark repressive modification induced by the PRC2 (11). ASXL1 also associates with the deubiquitinating enzyme BAP1, which may promote expression of genes (12)

Conflict of interest: The authors have declared that no conflict of interest exists. Citation for this article: JClin Invest. 2013;123(11):4627-4640. doi:10.1172/JCI70739. through removal of $\mathrm{H} 2 \mathrm{~A}$ lysine 119 ubiquitination placed by the PRC1 complex. Thus, ASXL1 appears to be involved in both PRC2mediated gene repression and opposition of PRC1 function (13).

Although loss of ASXL1 promotes myeloid transformation by impairing PRC2-mediated gene repression at a number of critical loci (11), intriguingly, most ASXL1 mutations are located in the 5 ' region of the last exon (exon 12), which are predicted to result in expression of a truncated ASXL1 protein. As further support for this, ASXL1 mutations are usually heterozygous, leaving 1 allele intact. Therefore, we hypothesized that the C-terminal truncated form of ASXL1 might function as a dominant-negative mutant that suppresses the ASXL1-WT function or alternatively as a gain-of-function mutant $(14,15)$. These possible effects of ASXL1 mutations have not been studied and are critical to delineate given the clinical importance of ASXL1 mutations.

In this study we show that ASXL1 mutations profoundly inhibited myeloid differentiation in vitro and induced typical MDS in a mouse model. We then sought to explore the molecular link between ASXL1 mutations and epigenetic disturbances that lead to development of MDS. We identify that expression of mutant forms of ASXL1 results in impaired PRC2 function and impaired myeloid differentiation in vitro and in vivo. Moreover, we identify that ASXL1 mutations induce upregulated expres- 
A

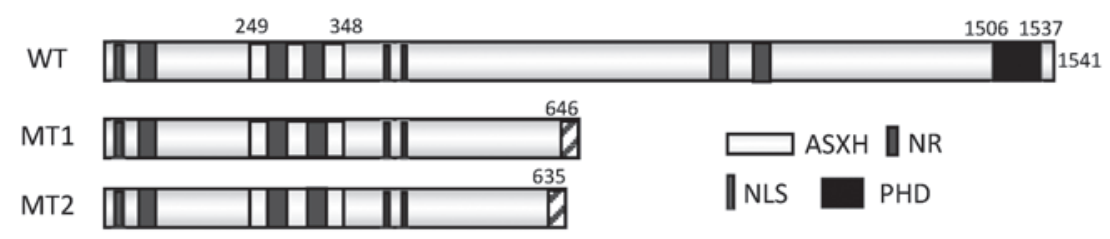

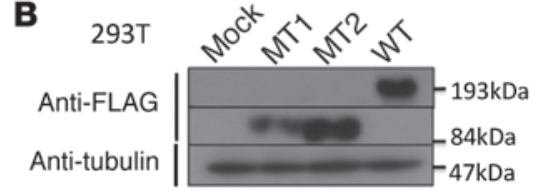

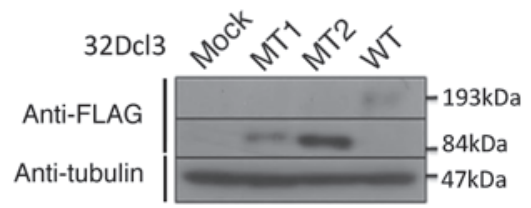

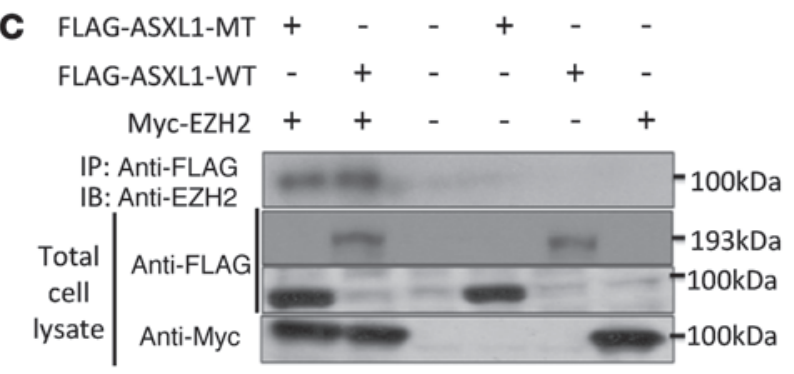

E
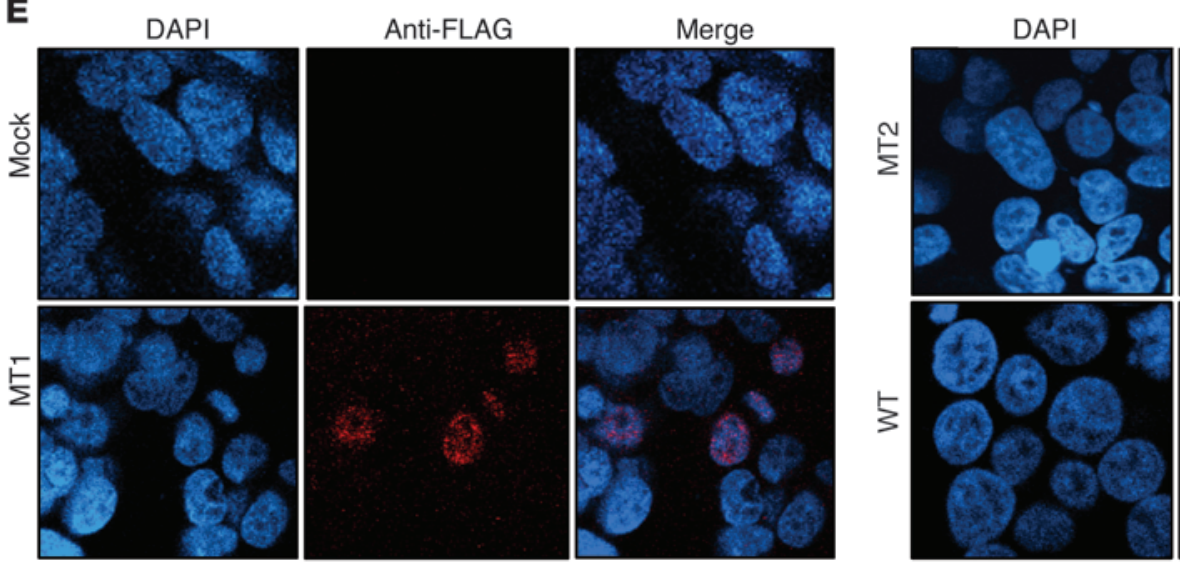

D

Anti-tubulin
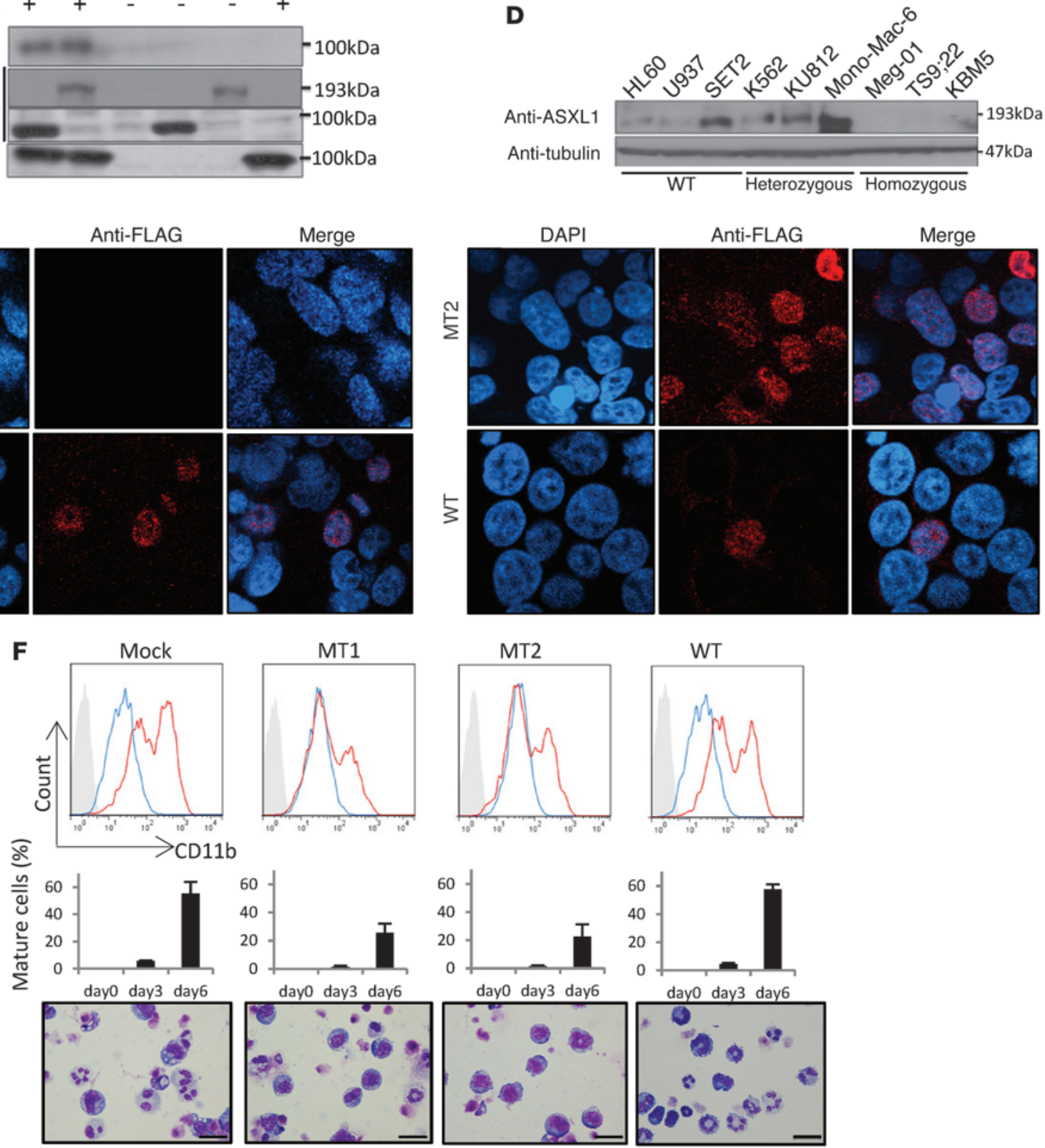


\section{Figure 1}

ASXL1 mutations inhibit G-CSF-induced myeloid differentiation of 32Dcl3 cells. (A) Schematic diagram of ASXL1-WT and 2 mutants, ASXL1-MT1 and ASXL1-MT2. ASXH, ASX homology region; NLS, nuclear localization signal; NR, putative nuclear receptor coregulator binding motifs; PHD, plant homeodomain. (B) Expression of ASXL1-WT and its mutants in 293T cells or 32Dcl3 cells transiently transfected or stably transduced with a FLAG-ASXL1-MT1, ASXL1-MT2, ASXL1-WT, or an empty vector (pMYs-IG). (C) HEK293T cells were transiently transfected with FLAG-ASXL1-MT, FLAGASXL1-WT, and Myc-EZH2 cDNA, followed by IP of FLAG epitope and Western blotting for EZH2. Cell lysates were also subject to immunoblotting with anti-FLAG Ab or anti-Myc Ab. (D) ASXL1 protein expression using C-terminus anti-ASXL1 antibodies in leukemia cell lines. Harboring mutations are as follows: K562, heterozygous ASXL1 Y591Y/X; KU812, heterozygous ASXL1 R693R/X; MonoMac-6, heterozygous ASXL1 L1393RfsX30; MEG-01, homozygous ASXL1 G646WfsX12; TS9:22, homozygous ASXL1 R693X; KBM5, homozygous ASXL1 G710X. (E) Nuclear localization of ASXL1-MTs. The 293T cells transiently transfected with pMYs-IG, pMYs-FLAGASXL1-MT1-IG, pMYs-FLAG-ASXL1-MT2-IG, or pMYs-FLAGASXL1-WT-IG were immunostained with anti-FLAG Ab (red) and DAPI (blue). Original magnification, $\times 600$. (F) Surface expression of CD11b in 32Dcl3 cells transduced with indicated plasmid after incubation with $1 \mathrm{ng} / \mathrm{ml} \mathrm{IL-3} \mathrm{(blue)} \mathrm{or} 50 \mathrm{ng} / \mathrm{ml} \mathrm{G-CSF}$ for 6 days (red) was analyzed by flow cytometry (top). Filled histograms show control (IgG). Proportions of segmented cells on days 0,3 , and 6 of G-CSF stimulation are shown (middle). Morphology of the cells 6 days after G-CSF stimulation was assessed by Wright-Giemsa staining (bottom). Original magnification, $\times 400$; scale bars: $20 \mu \mathrm{m}$.

sion of microRNA-125a (miR-125a) and subsequent suppression of C-type lectin domain family 5, member a (Clec5a), a type II membrane protein critical for myeloid differentiation. These results identify what we believe is a novel genetically accurate murine model of MDS and additional critical pathways for ASXL1-mediated myeloid transformation. Moreover, these findings suggest therapeutic potential for targeting the mutant forms of ASXL1 as well as miR-125 in the treatment of patients with myeloid malignancies.

\section{Results}

Mutant ASXL1 inbibited G-CSF-induced differentiation of $32 \mathrm{Dcl} 3$ cells. Most ASXL1 frame-shift mutations are found in the last exon, which are predicted to result in expression of C-terminal truncated forms. We constructed an N-terminal FLAG-tagged WT ASXL1 (FLAG-ASXL1-WT) as well as N-terminal FLAG-tagged truncated mutants of ASXL1 (FLAG-ASXL1-MT1 and -MT2 (Figure 1A). FLAG-ASXL1-MT1 and-MT2 were derived from the mutated genes of 1934dupG;G646WfsX12 and 1900-1922del;E635RfsX15, respectively, of patients with MDS. Although there is some controversy as to whether the most common ASXL1 mutation, 1934dupG;G646WfsX12, represents a true somatic mutation or an artifact (16), most studies have suggested this allele can occur as a somatic mutation in hematologic malignancies (17-19). When transiently expressed in $293 \mathrm{~T}$ cells or stably expressed in 32Dcl3 cells, these constructs expressed ASXL1-WT and ASXL1 mutant protein (ASXL1-MT) with expected molecular weights detected by an anti-FLAG antibody (Figure 1B). As reported previously (11), immunoprecipitation studies demonstrated that EZH2 bound ASXL1-WT. We further demonstrated that ASXL1-MT as well as ASXL1-WT can bind to EZH2 (Figure 1C). ASXL1-WT could also be detected in hemopoietic cell lines by anti-C-terminal ASXL1 antibodies (Figure 1D); HL60, U937, and SET2 harboring only the ASXL1-WT alleles expressed the WT $190-\mathrm{kDa}$ protein. In K562, KU812, and Mono-Mac-6 harboring 1 WT and 1 truncated mutant, ASXL1-WT could be easily detected with the C-terminal antibody. To detect C-terminal truncated endogenous ASXL1 proteins, we used antibodies against the N-terminal part of ASXL1. However, the backgrounds were so high that we could not determine whether the C-terminal-truncated ASXL1 was expressed. Similarly, in TS9;22, KBM-5, and MEG-01 cell lines harboring homozygous truncated ASXL1, we were not able to determine whether the truncated ASXL1 was expressed. We confirmed by quantitative real-time PCR (qRT-PCR) that the ASXL1 transcript was detected in these cell lines (Supplemental Figure 1; supplemental material available online with this article; doi:10.1172/JCI70739DS1). When ASXL1-MTs were expressed in $293 \mathrm{~T}$ cells, they localized to the nucleus (Figure 1E) similar to the ASXL1-WT, suggesting that the truncated ASXL1 mutant is expressed and prompting us to examine the effects of stable expression of ASXL1-MTs in both in vitro and in vivo experiments.

We investigated the effects of expression of WT and mutant ASXL1 alleles on hematopoietic differentiation. To this end, we expressed ASXL1-MT1 and-MT2 in 32Dcl3 cells and examined their effect on differentiation of the transduced cells. Interestingly, G-CSFinduced differentiation of $32 \mathrm{Dcl} 3$ cells was partially inhibited by the expression of ASXL1-MT1 and -MT2 but not by the expression of ASXL1-WT, based on morphology and flow cytometric expression of CD11b (Figure 1F). In addition, all-trans retinoic acid-induced (ATRA-induced) granulocytic differentiation of HL60 and GM-CSFinduced monocytic differentiation of FDC-P1 cells were attenuated by the overexpression of ASXL1-MT2 (Supplemental Figure 2A). The expression of ASXL1-MTs as well as ASXL1-WT did not affect the growth rate of $32 \mathrm{D}$ cells cultured in the presence of IL-3 and did not induce factor-independent growth (Supplemental Figure 2B). For further experiments, we mainly used ASXL1-MT2 unless otherwise specified, and refer to this mutant hereafter as ASXL1-MT.

ASXL1-MT reduced the expression of Clec5a/Mdl1. To elucidate the molecular mechanisms by which ASXL1-MT inhibited the G-CSFinduced differentiation of $32 \mathrm{Dcl} 3$ cells, we compared the expression profiles of parental 32Dcl3 cells and 32Dcl3 cells expressing ASXL1-MT maintained in the presence of $1 \mathrm{ng} / \mathrm{ml} \mathrm{IL-3} \mathrm{or} 50 \mathrm{ng} / \mathrm{ml}$ G-CSF. We identified a small set of genes whose expression levels changed more than 2-fold. These included Clec5a/Mdl1, Cd14, and Prom1. Expression of these genes was downregulated in 32Dcl3 cells expressing the ASXL1-MT. Among them, we focused on the type II transmembrane receptor Clec5a (20), because it was known that Clec5a expression is associated with granulocytic differentiation of $32 \mathrm{Dcl} 3$ cells (21). We confirmed that expression of Clec5a was reduced in 32Dcl3-expressing ASXL1-MT (Figure 2A). Moreover, while the expression of Clec5a in 32D cells was increased by G-CSF stimulation in a time-dependent manner, as previously reported (21), both in mRNA and protein levels, this increase was profoundly suppressed by the expression of ASXL1-MT but not by ASXL1-WT (Figure 2, A and B). This may result from the observation that myeloid differentiation of $32 \mathrm{Dcl} 3$ cells was inhibited by ASXL1-MT, and expression of Clec5a increases with myeloid differentiation, suggesting Clec5a serves as a marker of myeloid differentiation. On the other hand, ATRA or G-CSF-induced granulocytic differentiation of HL60 cells or 32Dcl3 cells, respectively, was enhanced by the expression of Clec5a (Figure 2C and 


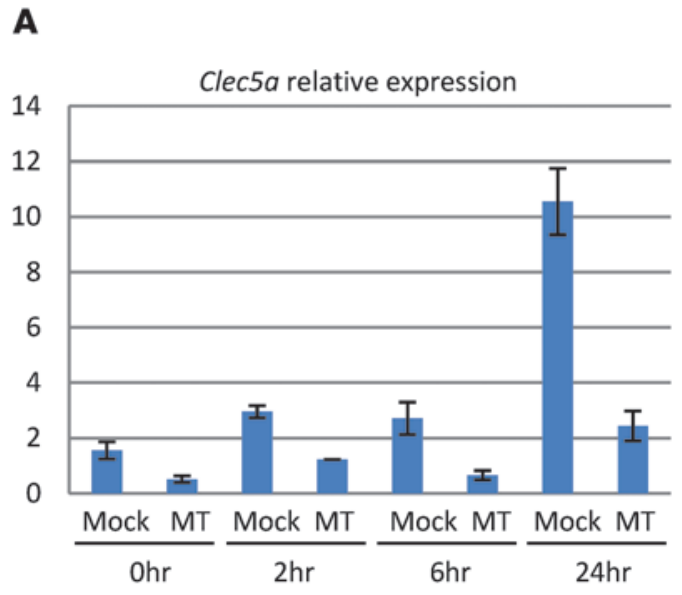

\section{C}

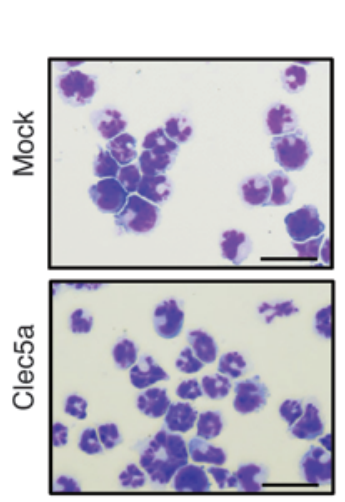

B
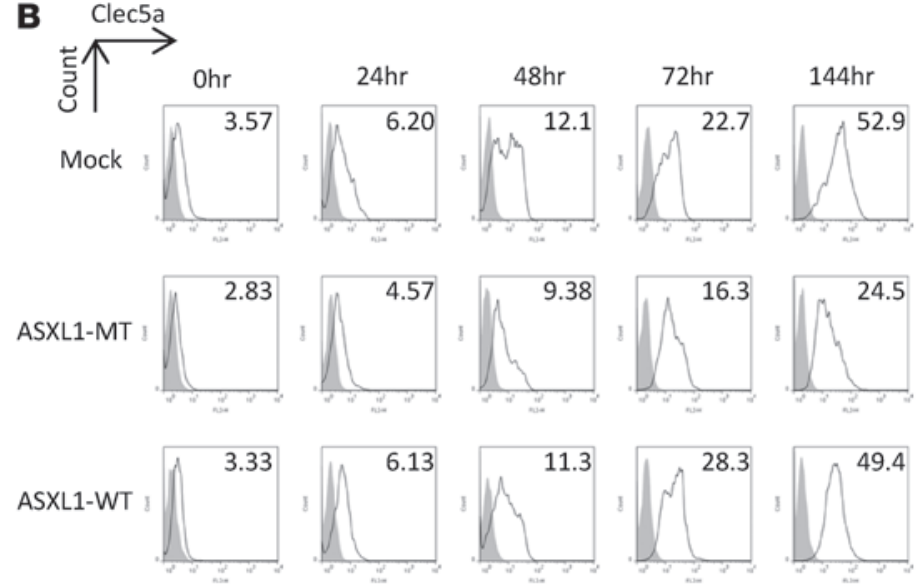

D

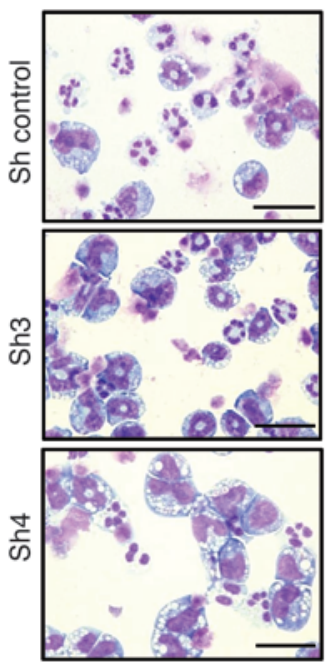

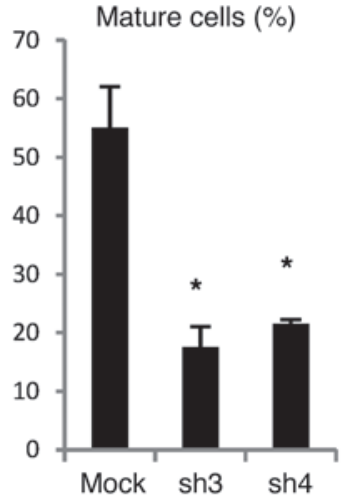

Figure 2

ASXL1 mutations reduced the expression of Clec5a, which contributed to the differentiation of 32Dcl3 cells. (A) qRT-PCR for Clec5a in 32Dcl3 cells transduced with pMYs-IG (mock) or pMYs-FLAG-ASXL1-MT2-IG (MT). Relative expression levels normalized by Gapdh mRNA were measured at the indicated time points after incubation with G-CSF $(50 \mathrm{ng} / \mathrm{ml})$. (B) Surface expression of Clec5a in 32Dcl3 cells transduced with mock, ASXL1-MT2 (MT), or ASXL1-WT after incubation with $50 \mathrm{ng} / \mathrm{ml} \mathrm{G-CSF}$. Cells were analyzed by flow cytometry at the indicated time points. MFIs are indicated. Filled histograms show control (IgG). (C) Left: Overexpression of Clec5a promoted the differentiation of HL60 cells. Morphology of HL60 cells expressing pMYs-IP (mock; top) and pMYs-Clec5a-IP (Clec5a; bottom) after incubation with 10-6 M ATRA for 3 days. Scale bars: $20 \mu \mathrm{m}$. Right: Proportion of segmented cells. (D) 32Dcl3 cells with or without shRNA for Clec5a were incubated with $50 \mathrm{ng} / \mathrm{ml}$ G-CSF for 6 days. Morphology (left) and proportion of differentiated 32Dcl3 cells (right) are shown. Scale bars: $20 \mu \mathrm{m}$. ${ }^{*} P<0.05$. Sh control, control scramble shRNA.

Supplemental Figure 3A), suggesting that Clec5a plays a direct role in the differentiation of myeloid cells.

To examine the direct involvement of Clec5a in G-CSF-induced differentiation of $32 \mathrm{Dcl} 3$ cells, we designed 2 shRNAs (sh3 and sh4) for Clec5a, which efficiently knocked down Clec5a expression in 32Dcl3 cells (Supplemental Figure 3, B and C). Intriguingly, G-CSF-induced differentiation of $32 \mathrm{Dcl} 3$ was significantly inhibited by Clec5a knockdown (Figure 2D), suggesting that ASXL1-MT inhibits differentiation of 32Dcl3 cells, at least in part through suppression of Clec5a. Importantly, ectopic expression of Clec5a in ASXL1-MT-expressing 32Dcl3 cells restored the differentiation ability of $32 \mathrm{Dcl} 3$, although this restoration was not induced by a mutant Clec5a that harbors a K16A mutation in the transmembrane domain, which disrupts the ability to associate with DAP12 (data not shown), an activating adaptor for
Clec5a that transmits the positive signal associating with Clec5a (Figure 3, A and B, Supplemental Figure 3D, and ref. 20). Thus, ASXL1-MT reduced the expression of Clec5a, which contributes to granulocytic differentiation of $32 \mathrm{Dcl} 3$ cells, leading to the disturbed differentiation of $32 \mathrm{Dcl} 3$ cells. Interestingly, expression of CLEC5A was reduced in the whole BM cells of the majority of patients with MDS when compared with normal BM cells (Figure 3C). However, the presence or absence of ASXL1 mutations did not correlate with CLEC5A expression in BM cells of MDS patients, suggesting that other MDS disease alleles can alter CLEC5A expression in patients with ASXL1-WT.

ASXL1-MTs induced MDS-like disease in mice. Using a mouse BM transplant (BMT) model, we next examined the in vivo effect of ASXL1-MT expression. BM cells derived from 5-fluorouracil-treated Ly5.2 mice were transduced with a retrovirus vector 
A

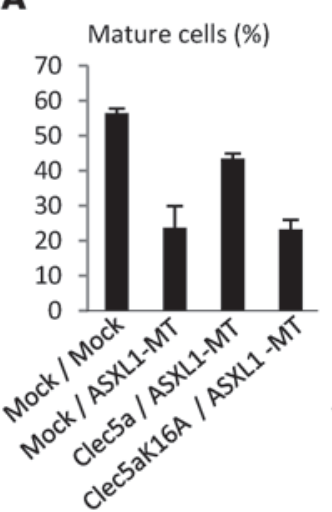

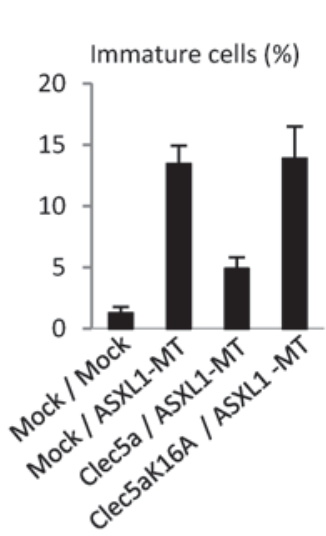

B
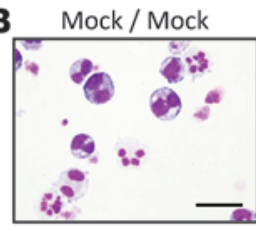

Clec5a / ASXL1-MT

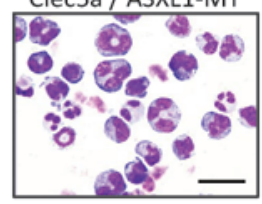

Mock / ASXL1-MT

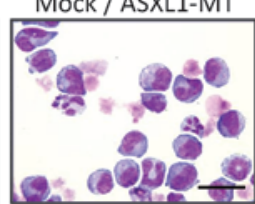

Clec5aK16A / ASXL1 -MT

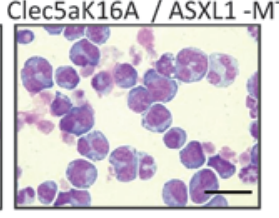

C CLEC5A expression relative to normal BM

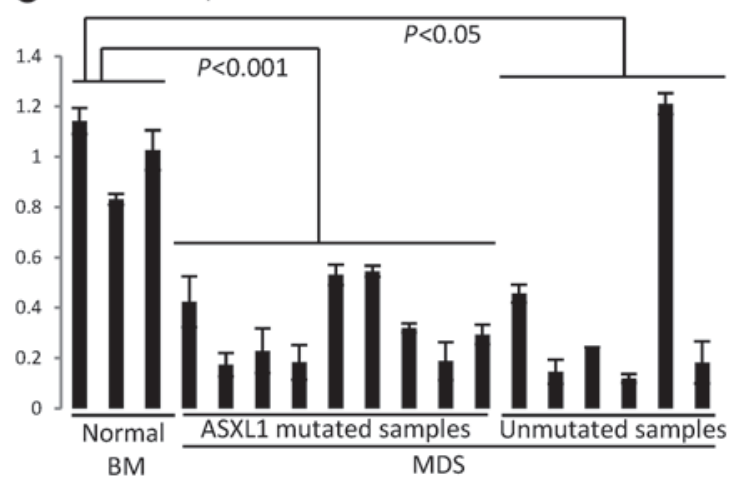

\section{Figure 3}

Downregulation of Clec5a caused by ASXL1-MT played a pivotal role in differentiation block. (A and B) 32Dcl3 cells transduced with $\mathrm{pMYs-IP/pMYs-}$ IB, pMYs-IP/ pMYs-ASXL1-MT2-IB, pMYs-Clec5a-IP/pMYs-ASXL1MT2-IB, and pMYs-Clec5a-K16A-IP/ pMYs-ASXL1-MT2-IB were cultured in the presence of $50 \mathrm{ng} / \mathrm{ml} \mathrm{G}-\mathrm{CSF}$ for 6 days. The proportions of mature or immature cells $(\mathbf{A})$ and cytospin preparations of these cells (B) are shown. Images were obtained with a BX51 microscope and an Olympus DP12 camera with a UplanFI objective lens. Original magnification, $\times 40$; scale bars: $20 \mu \mathrm{m}$. Data are representative of 3 independent experiments. (C) Relative expression levels of CLEC5A were examined by qRT$\mathrm{PCR}$ in whole BM cells derived from normal controls and from patients with ASXL1-mutated MDS and ASX$L 1$-WT MDS. The values were normalized by GAPDH mRNA levels. All data with error bars are presented as mean \pm SEM of 2 independent experiments. $P$ values were calculated using the 2-tailed Student's $t$ test or the Cochran-Cox test.
pMXs-FLAG-ASXL1-MT2-IG (IRES-GFP), and the transduced cells were transplanted into sublethally irradiated Ly5.1 mice. In the transplanted mice, the percentage of the GFP-positive cells gradually increased in the peripheral blood and reached $41 \%-100 \%$ one year after transplantation, while it gradually decreased over time in the mice transplanted with ASXL1-WT- or empty vectortransduced BM cells (Figure 4A). GFP-positive cells in the BM of ASXL1-MT-transduced mice 6 months after the transplantation were mostly CD11b positive and included relatively few B220positive cells, while GFP-negative, nontransduced cells consisted of equal numbers of CD11b-positive and B220-positive cells (Figure 4B). All of the transplanted mice displayed more or less morphological abnormalities 12 months or more after transplantation, mainly in myeloid cells and red blood cells (Figure 4C), including Pelger-Huet anomaly and hypersegmentation for myeloid cells and Howel-Jolly bodies, polychromasia, and anisopoikilocytosis for erythrocytes, consistent with multi-lineage dysplasia of human MDS. Mice expressing ASXL1-MT died of MDS after a long latency (median survival, 400.5 days), while most of the mock-transduced mice survived nearly 2 years without developing myeloid malignancies (Figure 4D). The GFP-positive BM cells that increased in ASXL1-MT-transduced mice were positive for CD11b and weakly positive for Gr1 and CD34. Expression of c-kit varied from positive to negative. These data suggest that a GFP-positive population contains immature and mature myeloid cells (Figure 4E). These mice developed severe anemia, leukopenia, and thrombocytopenia, while the BM was hypercellular and the spleen was enlarged (Figure 4, F and G). Thus, ASXL1-MT-transduced MDS mice developed pancytopenia with dysplasia in granulocytes and erythroid cells and occasionally progressed to overt leukemia, displaying all the features of human MDS. Overexpression of ASXL1-MT1 in the same BMT model revealed basically identical results (Supplemental Figure 4).

Expression profiles of hemopoietic cells of the mice that developed MDS. To elucidate the molecular mechanisms by which ASXL1-MT induced MDS, we performed expression profiles of BM cells of mice that developed MDS. We used CD3-B220-Ter119- BM cells of mock-transduced mice as a control. Gene set enrichment analysis (GSEA) indicated that ASXL1-MT induced an expression profile that inversely correlated with known PRC target genes (22), suggesting that ASXL1-MT inhibited PRC (Figure 5A). We also examined the expression of Hoxa genes and Clec5a in BM cells of the mice that developed MDS or MDS/AML after transplantation of ASXL1-MT-transduced BM cells; the expression of posterior Hoxa genes was increased and that of Clec $5 a$ was decreased in BM cells of MDS or MDS/AML mice when compared with BM cells of mock-transduced mice (Figure 5B). We next performed ChIP of the promoter regions of posterior Hoxa genes using $\mathrm{H} 3 \mathrm{~K} 27 \mathrm{me} 3$ antibodies and found that $\mathrm{H} 3 \mathrm{~K} 27 \mathrm{me} 3$ was greatly decreased around the promoter regions of Hoxa5, Hoxa9, and Hoxa10 in the MDS mice, correlating well with the upregulation of their mRNA expression (Figure 5, B and C). In addition, Western blot analysis of purified histones in 32Dcl3 cells transduced with ASXL1-MT indicated that $\mathrm{H} 3 \mathrm{~K} 27 \mathrm{me} 3$ was reduced globally (Figure 5D).

Interestingly, HOXA9 expression was increased in whole BM cells of most MDS patients harboring ASXL1 mutations, while the changes in HOXA9 expression was not significant in MDS patients without ASXL1 mutations (Figure 5E).

ASXL1-MT collaborated with N-Ras-G12V in inducing leukemia. ASXL1 knockdown reduces the latency and increases the severity 
A

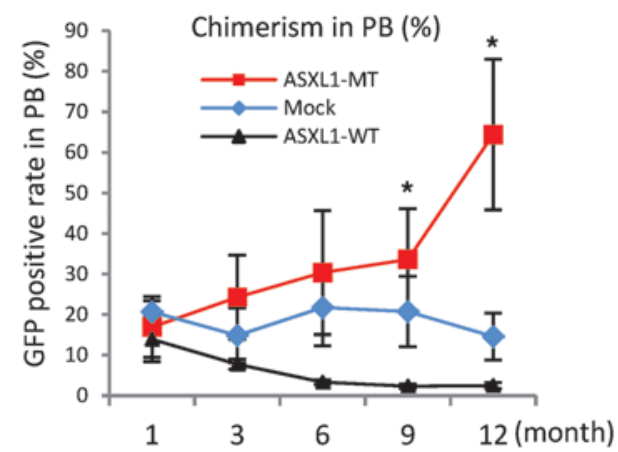

C
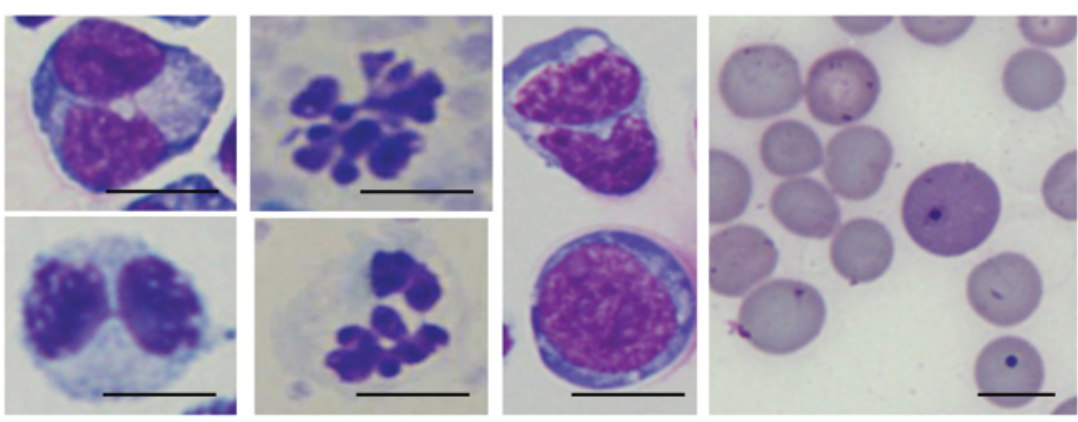

D

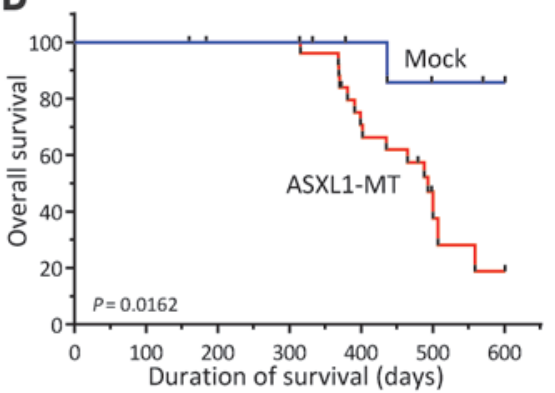

B
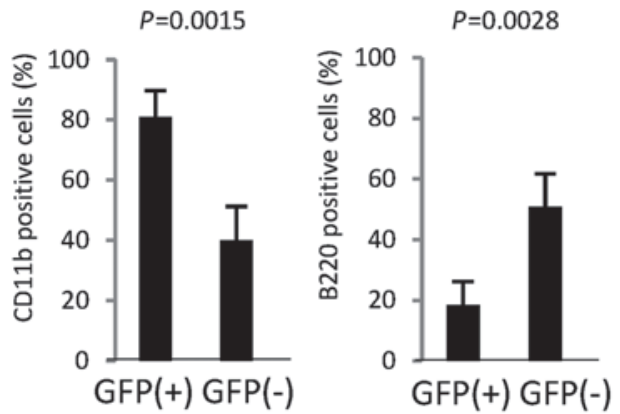

E
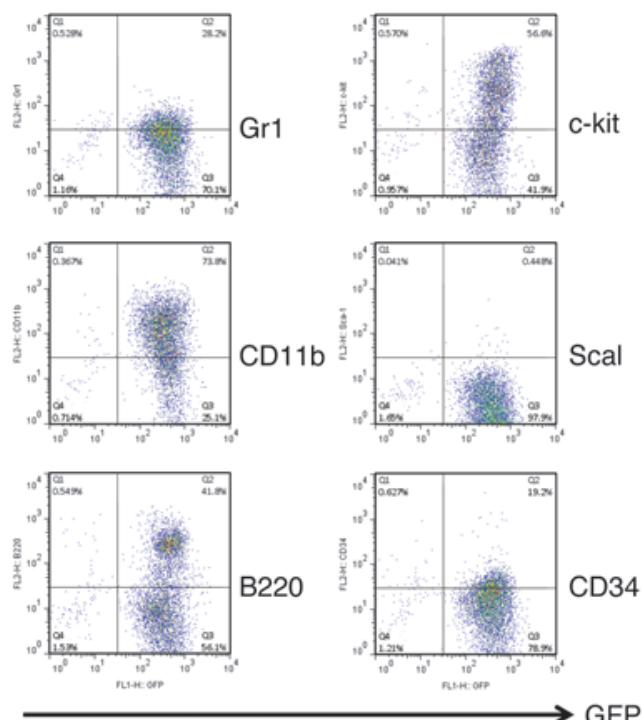
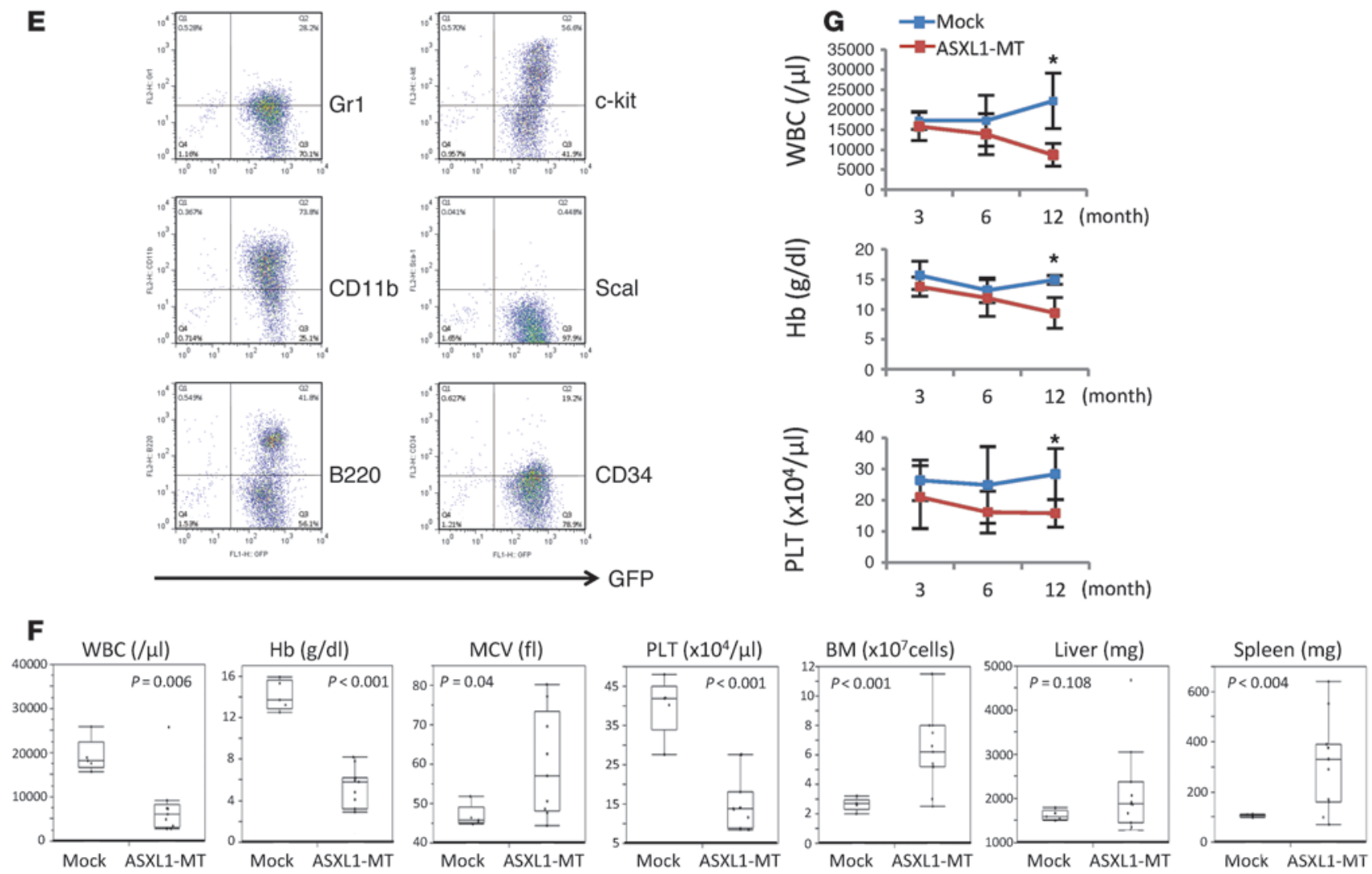


\section{Figure 4}

ASXL1 mutations induce MDS-like symptoms in a mouse BMT model. (A) Percentage of chimerism of donor cells in peripheral blood (PB). The chimerism of Ly5.1 donor-derived GFP-positive cells in $\mathrm{PB}$ (mean $\pm \mathrm{SEM}$ ) was examined after transplantation: mock, $n=6$; ASXL1-MT, $n=12$; ASXL1-WT, $n=6$. (B) Percentages of CD11bpositive or B220-positive cells related to GFP positivity in the BM of the transplanted mice determined by flow cytometric analyses. Samples were obtained from mice with pMYs-FLAG-ASXL1-MT2-IG, sacrificed 6 months after transplantation $(n=5)$. (C) Dysplasias of hematopoietic cells in mice receiving transplants of ASXL1 mutants were observed. Scale bars: $10 \mu \mathrm{m}$. (D) Kaplan-Meier analysis for the survival of mice that received transplants of BM cells transduced with pMYs-IG (mock, $n=13$, blue line) and pMYs-FLAG-ASXL1-MT2-IG (ASXL1-MT, $n=25$, red line). $P$ values were calculated using a log-rank test. (E) Flow cytometric analyses of BM cells derived from mice with ASXL1-MT. (F) Mice transplanted with ASXL1-MT2 (ASXL1-MT, $n=11$ ) displayed progressive pancytopenia, macrocytosis, and hyperplastic $\mathrm{BM}$ and increased splenomegaly compared with mice transplanted with empty vector (mock, $n=5$ ). BM cells were isolated from the femurs and tibias of the sacrificed mice. (G) Blood count data, including white blood cells (WBC), hemoglobin ( $\mathrm{Hb}$ ), and platelets (PLT), at 3,6 , or 12 months after transplantation are indicated (mean \pm SEM). Mock, $n=6$; ASXL1-MT, $n=12 .{ }^{*} P<0.05$.

of myeloproliferation induced by N-Ras-G12D (11). We thus examined whether stable expression of ASXL1-MT might similarly collaborate with N-Ras-G12V in vivo. As reported, N-RasG12V induced MPN-like diseases in the BMT model (23). In this model, co-transduction of ASXL1-MT, but not ASXL1-WT, with $\mathrm{N}$-Ras-G12V significantly shortened latency when compared with mice transplanted with BM cells transduced with N-Ras-G12V alone (Figure 6A and data not shown) and increased the number of immature blast cells (35\% vs. $15 \%$ ), indicating that the combination of N-Ras-G12V and ASXL1-MT induced progression to AML rather than MPN (Figure 6, B and C). It also enhanced hepatosplenomegaly in the affected mice (Figure 6, D and E), while hemoglobin concentrations and number of white blood cells did not change significantly (Figure 6F). The expression of Hoxa9 was increased in leukemic cells induced by ASXL1-MT and $\mathrm{N}$-Ras-G12V when compared with that in MPN cells induced by N-Ras-G12V alone (Figure 6G), suggesting that ASXL1-MT inhibited PRC2-driven repression of transcription. On the other hand, expression of Clec5a was significantly decreased in leukemic cells induced by ASXL1-MT and N-Ras-G12V (Figure 6G). Other surface markers of MPN or AML cells induced by N-Ras-G12V or N-Ras-G12V and ASXL1-MT, respectively, were similar, although expression of CD11b and c-kit was slightly higher in N-Ras-G12V and ASXL1-MT-induced AML compared with N-Ras-G12Vinduced MPN (Figure 6H). Thus, ASXL1-MT accelerated the onset of MPN induced by N-Ras-G12V and resulted in moderate increase in immature blasts and leukemic transformation, with increased expression of Hoxa9 and decreased expression of Clec5a.

miR-125a is responsible for the repression of Clec5a by ASXL1-MT. As shown in Figure 5D, H3K27me3, a transcriptionally repressive mark, was globally reduced by ASXL1-MT. In order to ascertain whether a miRNA involved in myeloid transformation might be upregulated with ASXL1 mutations, we performed microarray analysis of miRNAs in ASXL1-MT-transduced BM cells and mock-transduced BM cells of the BMT model. We identified increased expression of several miRNAs in BM cells expressing
ASXL1-MT, including miR-671, miR-125a, miR-714, miR-18b, miR-129, and miR-3107 (Supplemental Table 1). Among these, miR-125a is known to regulate hematopoietic stem cell numbers and induce MPN in a mouse BMT model $(4,24)$. A closely related miRNA, miR-125b, induces a variety of hematologic malignancies in transgenic mice and BMT models (25-27). We confirmed that expression of miR-125a was increased in 32Dcl3 cells expressing ASXL1-MT when compared with those expressing ASXL1-WT or the empty vector (Supplemental Figure 5). We next evaluated whether Clec5a is a target gene of miR125a. We identified a recognition site of miR-125a in the $3^{\prime}$ untranslated region ( $3^{\prime}$ UTR) of mouse Clec5a and 2 recognition sites in the $3^{\prime} \mathrm{UTR}$ of human CLEC5A (Figure 7A). Therefore, we focused on Mus musculus miR-125a (mmu-miR-125a) and further investigated the effects of miR-125a expression.

First, to confirm that the $3^{\prime} \mathrm{UTR}$ of the murine Clec5a gene was targeted by miR-125a, we generated an EF1 $\alpha$ promoter-driven luciferase construct harboring the $3^{\prime}$ UTR of the Clec 5 a gene (Figure 7B) and performed a luciferase assay. As expected, the presence of the $3^{\prime}$ UTR of the Clec5a gene reduced luciferase activity (Figure 7C). This reduction was completely cancelled by mutating the miR-125a target sequence in the 3'UTR of the Clec5a gene (Figure 7, B and C). miR-125b1 and miR-125b2 are genes distinct from miR-125a, but their seed sequence is identical with that of miR-125a. When miR-125a or miR-125b was expressed in 32Dcl3 cells, Clec5a expression was reduced both at mRNA levels and surface expression levels (Figure 7, D and E) compared with 32Dcl3 cells transduced with empty vector. Moreover, these cells became more resistant to G-CSF-induced differentiation (Figure 7F). These results clearly demonstrate that miR-125a targets Clec5a expression, leading to the inhibition of differentiation.

We next examined H3K27me3 near the transcription start site (TSS) of miR-125a in 32Dcl3 cells transduced with the empty vector, ASXL1-MT, or ASXL1-WT. This revealed profound reduction of H3K27me3 near the TSS of miR-125a in 32Dcl3 cells expressing ASXL1-MT (Figure 8, A-C). Moreover, H3K27me3 near the TSS of miR-125a was also reduced in BM cells of the mice that developed MDS after the transplantation of the ASXL1-MT-transduced BM cells, when compared with cells from mice engrafted with control BM cells (Figure 8, A, D, and E). Intriguingly, EZH2 binding to the miR-125a locus was reduced in ASXL1-MT-expressing 32Dcl3 cells or in ASXL1-MT-transduced BM cells from mice that developed MDS, while ASXL1-WT seemed to increase EZH2 binding to the miR-125a locus (Figure 8, B and D). Altogether, these results indicated a scenario in which ASXL1-MT hampered EZH2 binding to the miR-125a locus and suppressed EZH2-mediated H3K27me3 near the TSS of the miR-125a gene, leading to derepression of miR-125a. The increased miR-125a expression inhibited the expression of Clec $5 a$, contributing to the impaired differentiation of mouse BM cells that developed MDS.

\section{Discussion}

We have established and characterized a mouse MDS model induced by ASXL1 mutations. Although MDS patients frequently harbor multiple somatic mutations, it is not clear whether combinations of multiple mutations are required for the development of MDS. In addition, the molecular mechanisms of MDS pathogenesis remain elusive, particularly for recently identified mutations in epigenetic factors. Here we demonstrate that expression of the ASXL1-MT alone induces MDS in the mouse BMT model after a 
A Enrichment score (ES): 0.4093917 NES: 1.8348485

FDR q-value: 0.0012032641

Enrichment plot: KIM PRC MODULE HUMAN ORTHOLOGS

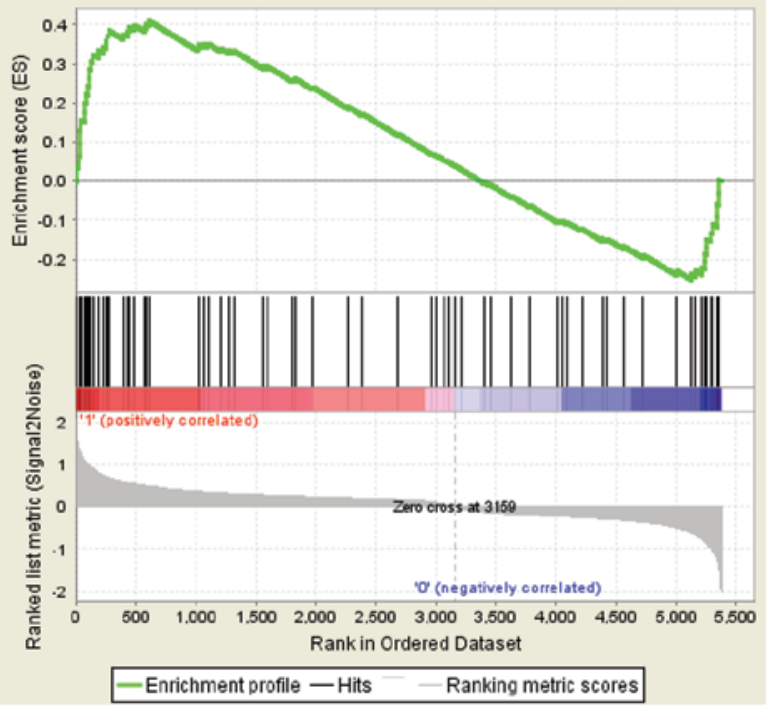

D

$32 \mathrm{Dcl} 3$ cells

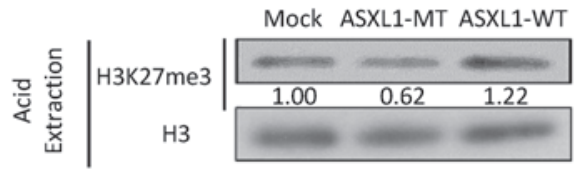

E

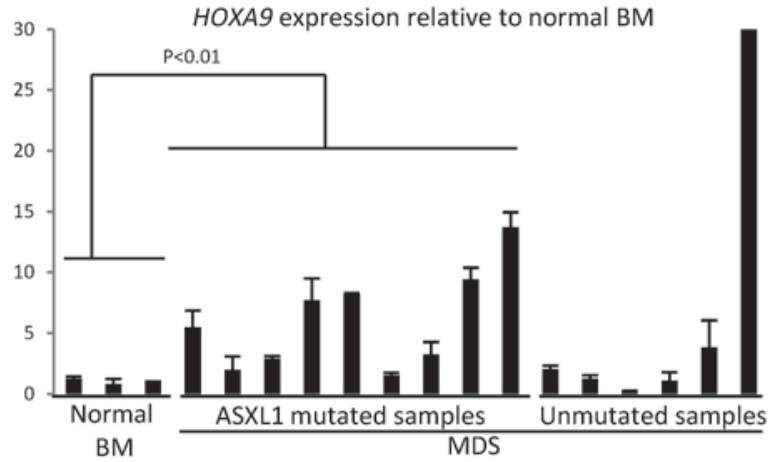

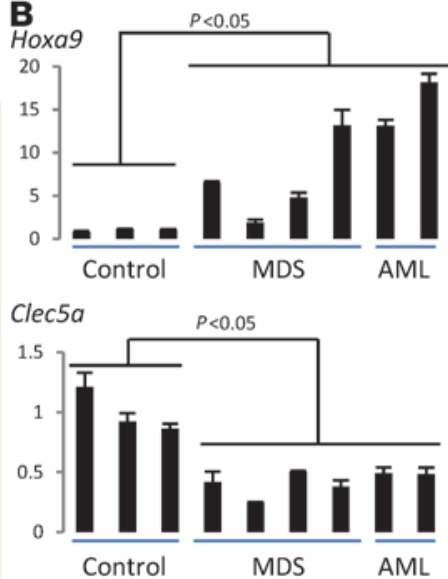
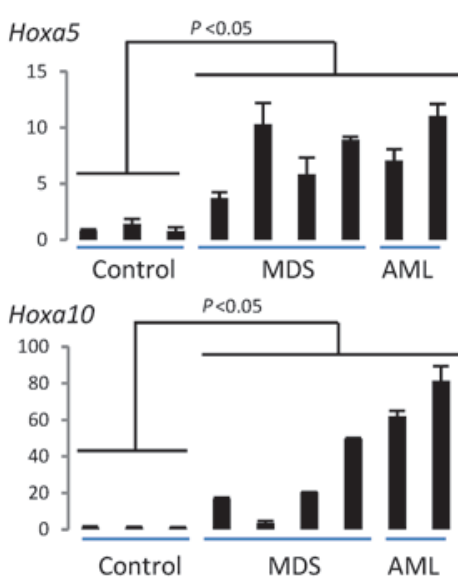

C
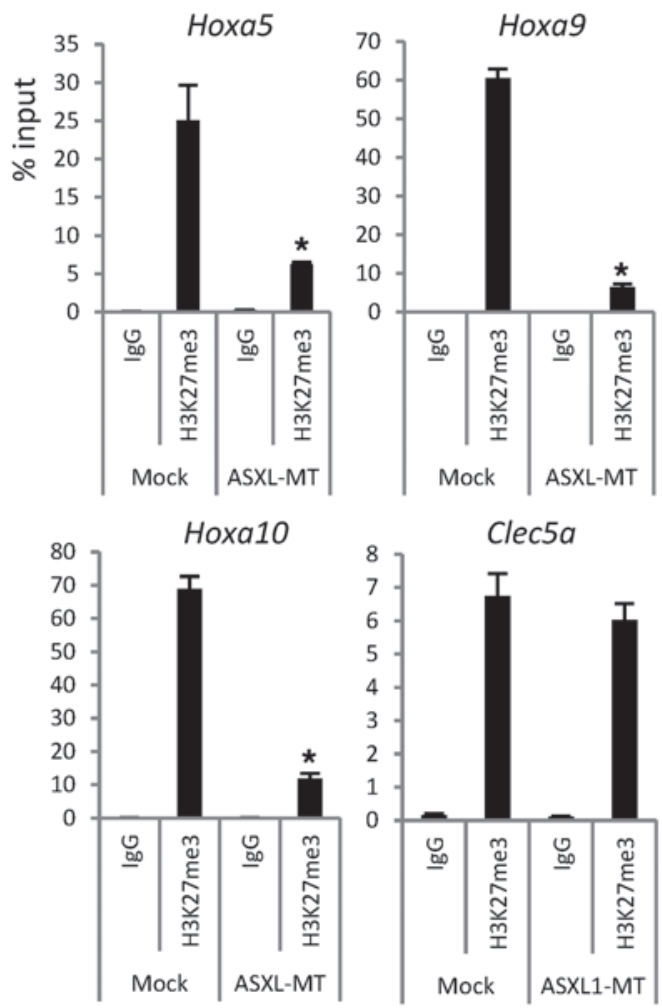

Figure 5

ASXL1-MT inhibited EZH2 functions, leading to loss of H3K27me3 at the HoxA locus. (A) GSEA of microarray analysis of mice with ASXL1-MT revealed a significant enrichment of genes found in a previously described gene expression signature of the PRC target, compared with that of mice with empty vectors. (B) In the mouse BMT model, ASXL1-MT-induced MDS/AML resulted in increased Hoxa5, Hoxa9, and Hoxa10 and decreased Clec5a mRNA expression as shown by qRT-PCR analysis in BM cells from transplanted mice. (C) ChIP for H3K27me3 followed by qPCR across the Hoxa5, Hoxa9, Hoxa10, and Clec5a locus in BM cells of mice that received transplants of BM cells transduced with pMYs-IG (mock) or pMYs-FLAG-ASXL1-MT-IG (ASXL1-MT). (D) Acid-extracted histones were obtained from 32Dcl3 cells transduced with pMYs-IG, pMYs-FLAG-ASXL1-MT-IG, and pMYs-FLAG-ASXL1-WT-IG, and then analyzed by Western blotting using anti-H3K27me3 antibodies. Levels of histone modifications were normalized to the amount of histone $\mathrm{H} 3$ and are indicated using ImageJ. (E) Relative expression levels of $H O X A 9$ were examined by qRT-PCR in whole BM cells derived from normal controls and from patients with ASXL1-mutant MDS and ASXL1-WT MDS. The values were normalized by GAPDH mRNA levels.

long latency, indicating that ASXL1-MT is a driver mutation in MDS development. Mutations in ASXL1 have been reported in patients with the entire spectrum of myeloid malignancies and are recurrently associated with adverse overall survival, independent of conventional clinical predictors $(8,28)$; this highlights a critical need to understand the precise mechanism of transformation by ASXL1 mutations. Recently, it was indicated that a subset of ASXL1 mutations result in loss of stable expression of full-length ASXL1 
A
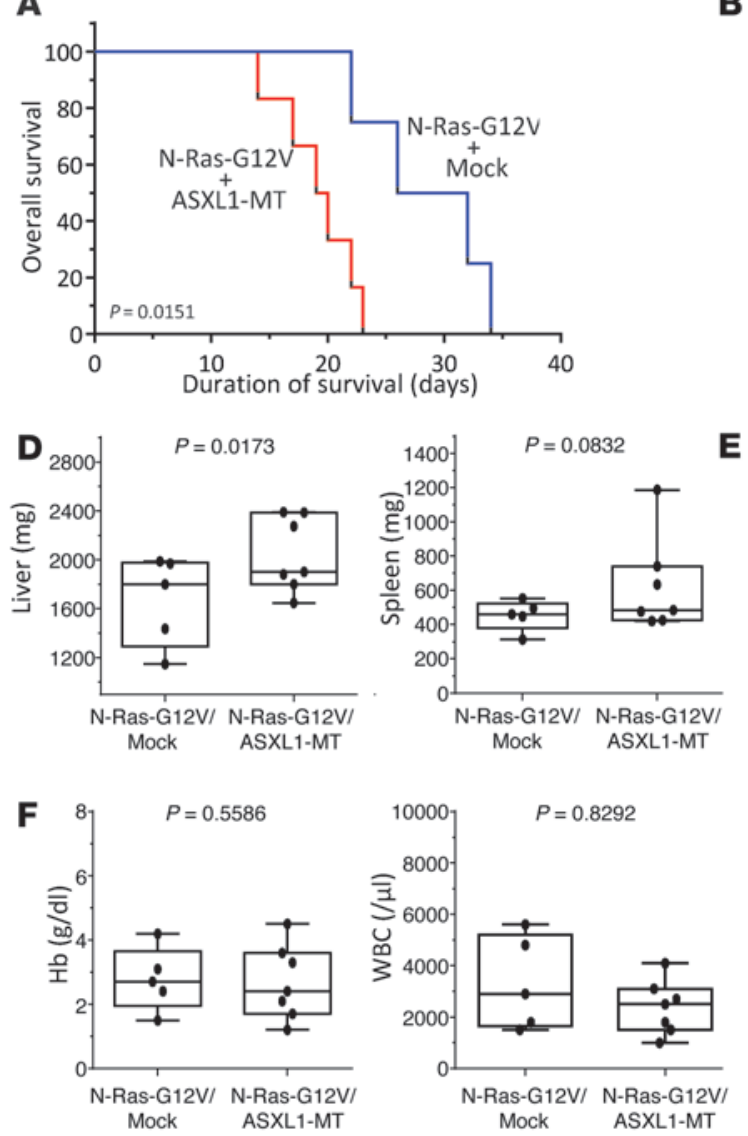

G

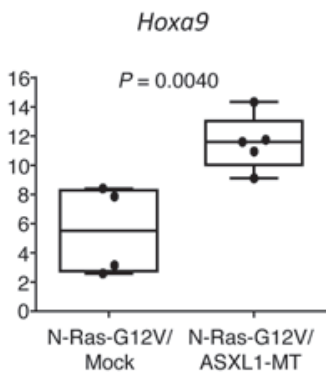

B

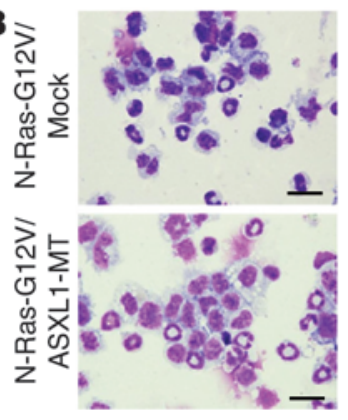

E

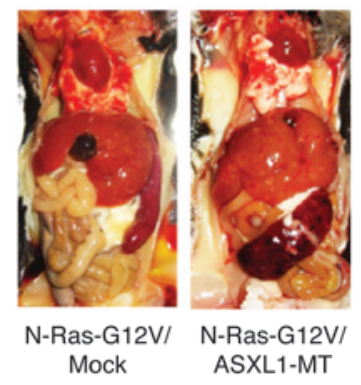

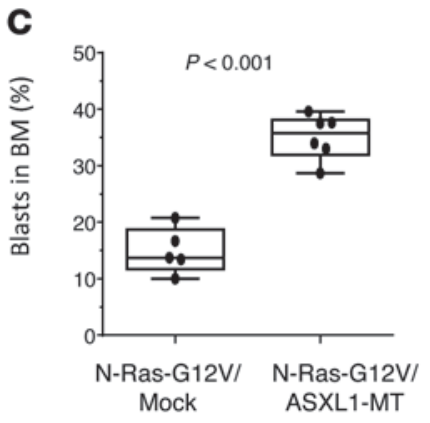

H
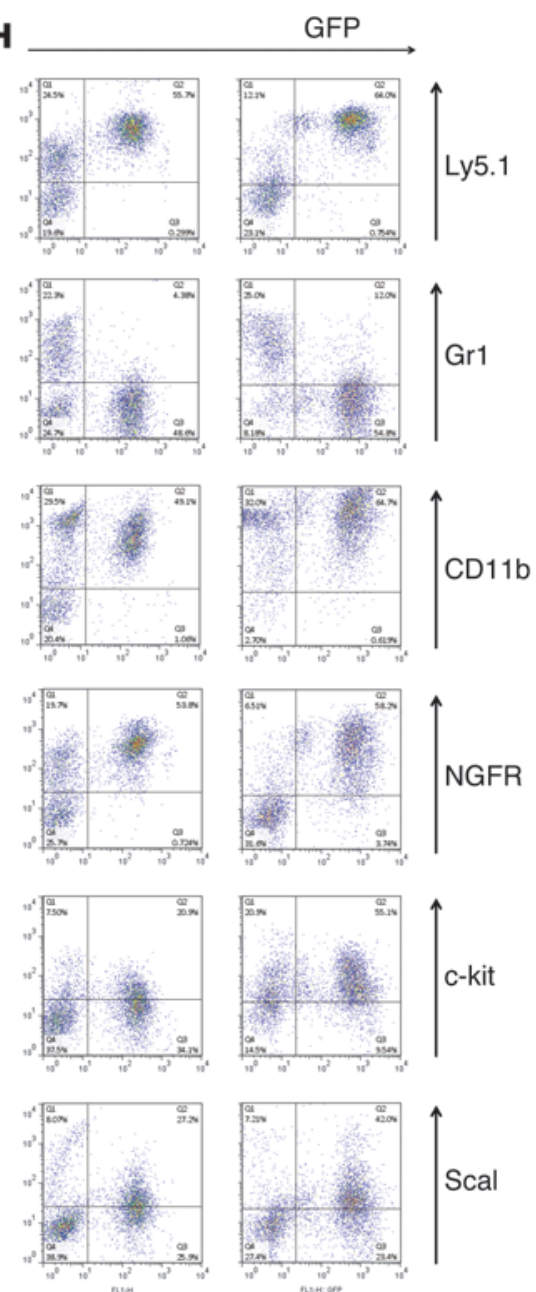

N-Ras-G12V/

Mock

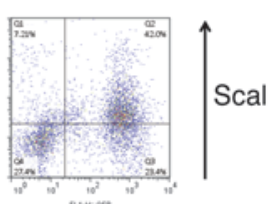

N-Ras-G12V/

\section{Figure 6}

ASXL1 mutations collaborated with the N-Ras activating mutation in inducing myeloid leukemia. (A) Kaplan-Meier analysis for the survival of mice that received transplants of BM cells transduced with pMYs-N-Ras-G12V-IG and pMYs-INGFR (N-Ras-G12V + mock, $n=4$, blue line) and pMYs-N-Ras-G12V-IG and pMYs-ASXL1-MT2-INGFR (N-Ras-G12V + ASXL1-MT, $n=6$, red line). $P$ values were calculated using a log-rank test. (B) Cytospin preparations of BM cells derived from mice transplanted with N-Ras-G12V + mock and N-Ras-G12V + ASXL1-MT were stained with Giemsa. Representative photographs are shown. Original magnification, $\times 400$; scale bars: $20 \mu \mathrm{m}$. (C and D) Mice transplanted with N-RasG12V/ASXL1-MT displayed increased numbers of leukemic blasts in BM (C) and hepatomegaly and splenomegaly (D), although the differences in spleen weight were not statistically significant. $P$ values were calculated using the Student's $t$ test (C) or Cochran-Cox test (D). (E) Macroscopic findings of sacrificed mice transplanted with BM cells transduced with the indicated construct. Representative photographs are shown. (F) Mice transplanted with N-Ras-G12V/mock and N-Ras-G12V/ASXL1-MT displayed severe anemia and leukopenia to a similar extent. (G) qRT-PCR revealed an increased expression of Hoxa9 and decreased expression of Clec5a in the BM of mice transplanted with N-Ras-G12V/ASXL1-MT. $P$ values were calculated using the Cochran-Cox test. $(\mathbf{H})$ Flow cytometric analysis of BM cells derived from sacrificed mice transduced with indicated constructs. 
A

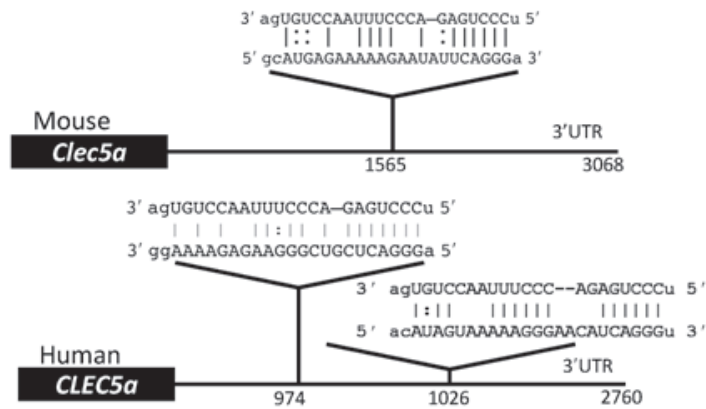

B

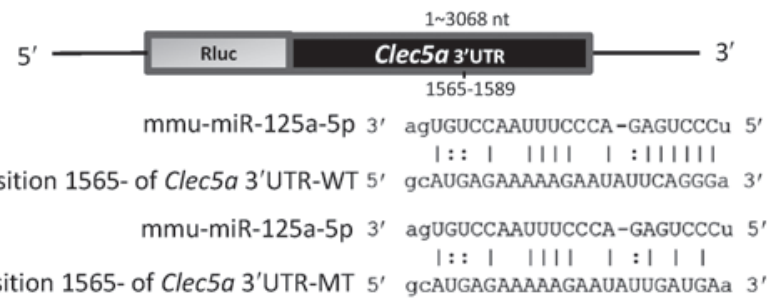

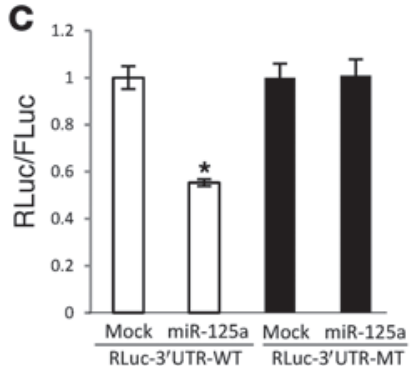

D

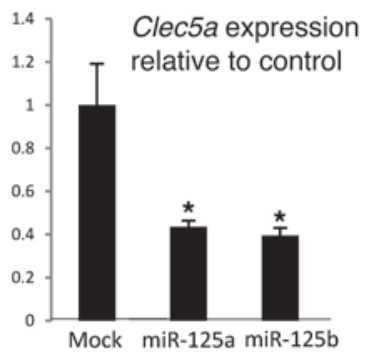

$\mathbf{E}$

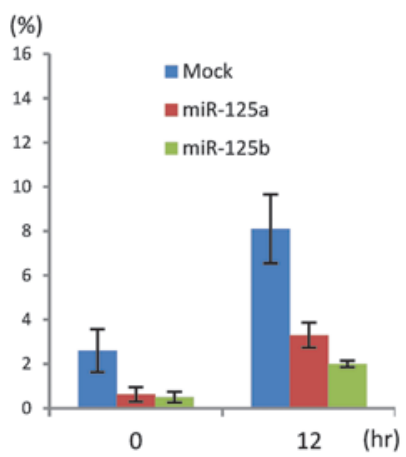

F $(\%)$

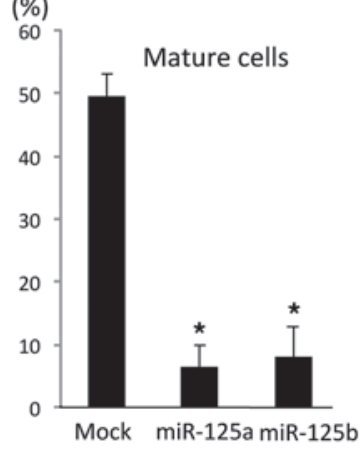

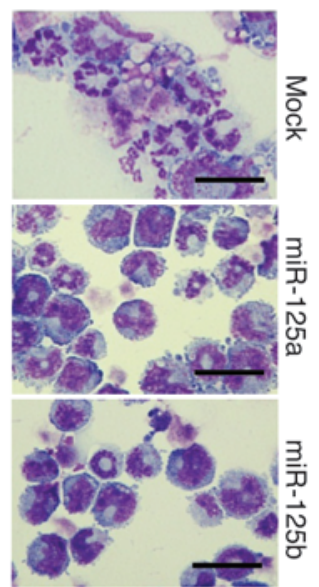

Figure 7

ASXL1 mutations caused upregulation of miR-125a, leading to the repression of Clec5a. (A) Schematic presentation of predicted miR125a-binding sites in the mouse Clec5a (top) and human CLEC5A 3'UTR (bottom). (B) To confirm that Clec5a is a direct target gene of miR-125a, the WT 3'UTR of Clec5a or the mutated 3'UTR was cloned to downstream of the Renilla luciferase (Rluc) open reading frame. Schematic diagrams of predicted miR-125a-binding sites in the Clec5a-3'UTR and the alignment between miR-125a and either Clec5a-3'UTR (top) or a mutated 3'UTR (bottom) are shown. Three bases in the 3'UTR, corresponding to seed sequences, were replaced with the indicated bases in the mutant form. (C) 293T cells were cotransfected with an internal control vector (pGL3-control) plus either pGL4.74[hRluc/TK]-Clec5a3'UTR-WT or pGL4.74[hRluc/TK]-Clec5a3'UTR-MT plus either pMXs-EF1-miR-125a-Puro or mock (pMXs-EF1-Puro). Luciferase assays were performed with a triplicate set. (D) Relative expression levels of Clec5a by qRT-PCR in 32Dcl3 cells transduced with pMXs-EF1-Puro (mock), pMXs-EF1-miR-125a-Puro, and pMXs-EF1-miR-125b-Puro. (E) Positive rate of Clec5a expression in 32Dcl3 cells transduced with mock, miR-125a, or miR-125b after incubation with $1 \mathrm{ng} \mathrm{IL-3}$ ( 0 hours) or $50 \mathrm{ng} / \mathrm{ml}$ G-CSF ( 12 hours) was analyzed by flow cytometry at indicated time points. (F) The proportion of mature cells (left) and cytospin preparations (right) of the 32Dcl3 cells expressing mock, miR-125a, or miR-125b cultured in the presence of $50 \mathrm{ng} / \mathrm{ml} \mathrm{G}-\mathrm{CSF}$ for 6 days. Scale bars: $20 \mu \mathrm{m} .{ }^{*} P<0.05$.

and that depletion of ASXL1 promoted myeloid transformation through impaired PRC2-mediated H3K27 methylation (11).

ASXL1 mutations mostly occur as heterozygous mutations, and the mutations conspicuously occur as nonsense and frameshift mutations in the last exon, prior to the PHD domain. This suggests that most ASXL1 mutations result in a stable protein product that may have gain-of-function mutations. Here we identify that stable expression of ASXL1-MT, which results in global downregulation of $\mathrm{H} 3 \mathrm{~K} 27 \mathrm{me} 3$, impaired myeloid differentiation and collaborated with co-occurring genetic alterations, as was seen with downregulation of ASXL1-WT. The observation that expression of ASXL1-MT mirrors the effects of downregulation of ASXL1-WT strongly suggests that in addition to known loss-of-function mutations, a subset of ASXL1 mutations confer dominant-negative activity.

Expression of ASXL1-MT as demonstrated here resulted in impaired myeloid differentiation in vitro and in vivo. ASXL1 
A

mmu-miR-125a

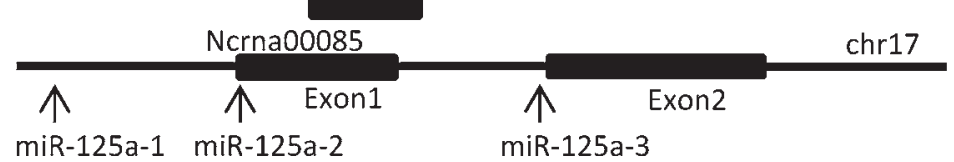

B

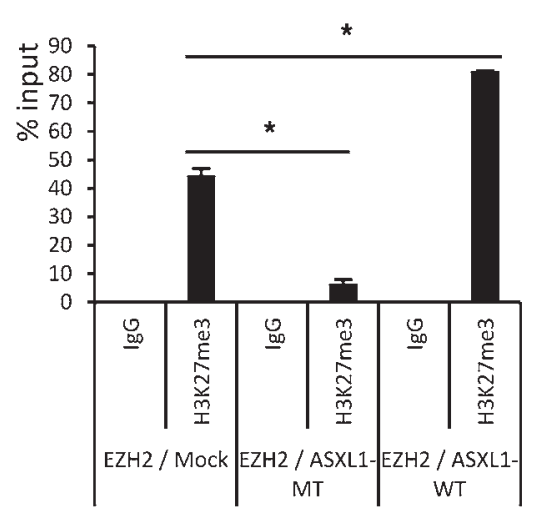

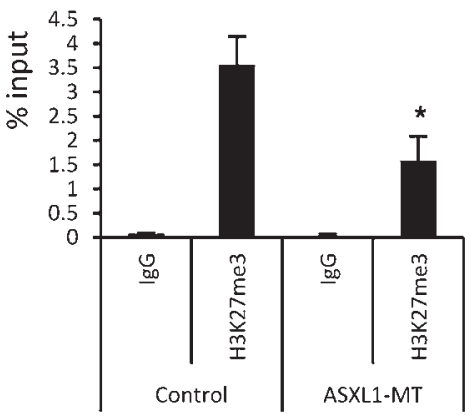

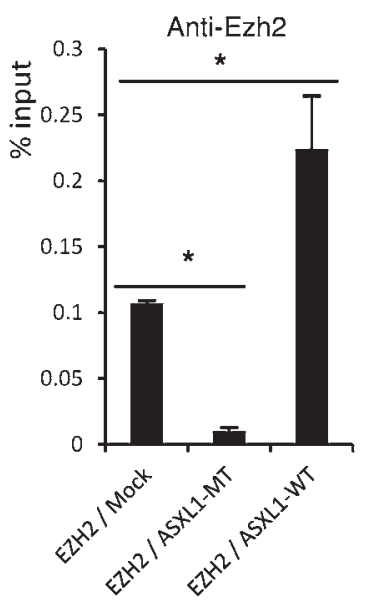

Anti-Ezh2

c
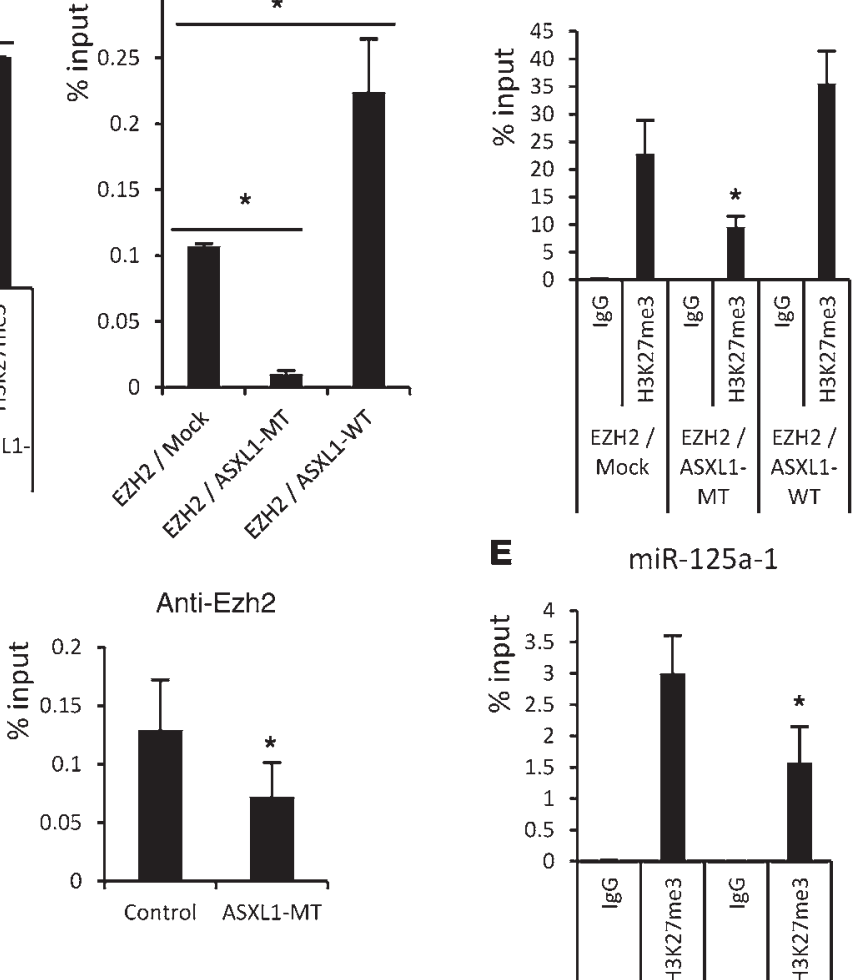

E

miR-125a-1

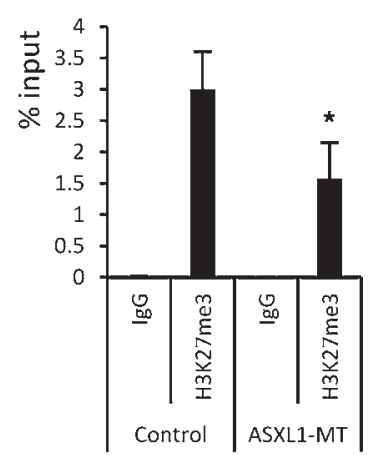

miR-125a-3

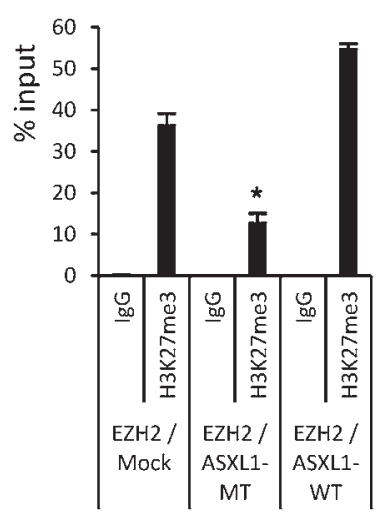

miR-125a-3

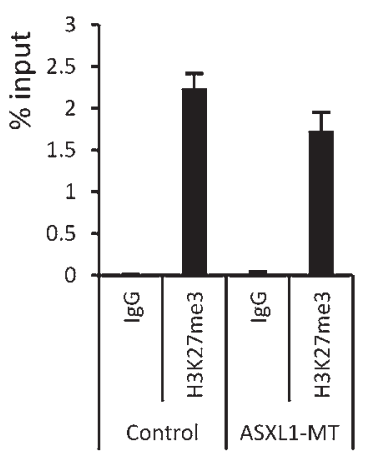

Figure 8

ASXL1 mutations caused loss of H3K27me3 at the miR-125a locus. (A) Schematic diagram of the mmu-miR-125a and Ncrna00085 loci indicating their genomic structures. Exons are indicated by black boxes. Regions amplified from the precipitated DNA by site-specific quantitative PCR are indicated by arrows. (B-E) Quantitative ChIP analyses of 32Dcl3 cells transduced with pMYs-EZH2-IG and pMYs-IP (EZH2/mock), pMYs-EZH2IG and pMYs-FLAG-ASXL1-MT2-IP (EZH2/ASXL1-MT), or pMYs-EZH2-IG and pMYs-FLAG-ASXL1-WT-IP (EZH2/ASXL1-WT) (B and C) and BM cells from the mice transplanted with BM cells transduced with pMYs-IG (Mock) or pMYs-FLAG-ASXL1-MT2 (ASXL1-MT) (D and E). Abs specific to H3K27me3 or Ezh2 and primers for miR-125a-2 (B and D) and for miR-125a-1/3 (C and E) were used for ChIP analyses. There were no detectable or very low levels of background signals with IgG isotype controls at all amplified regions. Percentages of input DNA are shown as the mean \pm SEM for duplicate analyses. ${ }^{*} P<0.05$. Data are representative of 3 independent experiments.

mutations are most frequent in patients with MDS, MDS/MPN overlap syndromes, and AML with myelodysplasia-related changes, highlighting a close relationship between impaired myeloid differentiation and ASXL1 mutations (29). We identified that expression of ASXL1-MT in vivo results in the development of a lethal disorder characterized by morphologic dysplasia, leukopenia, and impaired myeloid differentiation with subsequent transformation to AML in some cases, all hallmarks of human MDS. Moreover, co-expression of mutant forms of ASXL1 with oncogenic N-RAS, a complex genotype common to patients with CMML (10), results in a lethal myeloid malignancy with shorter latency than that seen with oncogenic N-RAS alone. Given the paucity of genetically accurate murine models of MDS, expression of ASXL1-MT as demonstrated here may be quite valuable for further mechanistic and preclinical studies of MDS and AML with myelodysplasia-related changes. Interestingly, it has been recently reported that disruption of the ASXL1-interacting molecule BAP1 in mice induced CMML-like disease (30). BAP1 mutation was also identified in a patient with MDS, implicating the BAP1-ASXL1 axis in suppressing MDS. The contribution of disruption of the ASXL1-BAP1 axis (PRC1 related), versus loss of H3K27 trimethylation (PRC2 related), in promoting myeloid transformation induced by ASXL1-MT requires further evaluation.

To understand the transcriptional events responsible for the biological effects of ASXL1 mutations in more detail, we performed transcriptomic studies in the presence of mutant ASXL1 in vitro and in vivo. In addition to identifying significant upregulation of posterior Hoxa genes, we identified increased expression of miR-125a due to locus-specific downregulation of H3K27 methylation. Moreover, it has been reported that miR-125a is upregulated after inhibition of EZH2 by knockdown 
in DU145 prostate cancer cells (31). miR-125a was the only miR upregulated in both our murine system and in primary human MDS samples (32). miR-125a belongs to the miRNA family that also includes miR-125b1 and miR-125b2, all of which have the same seed sequence and have been implicated in human leuke$\mathrm{mia} /$ lymphoma (33-35) as well as in MDS (32). Forced expression of these miRs has been previously shown to induce the expansion of hemopoietic stem cells or hamper myeloid differentiation (4, 24-26, 32, 35).

Although several proapoptotic genes have been implicated for hematopoietic transformation as miR-125a targets $(4,26,36)$, here we identified direct repression of a novel target Clec $5 a$ by miR-125a as a specific effector of the differentiation phenotype. The type II membrane protein CLEC5A (20) belongs to the lectin family and associates with the adaptor protein DAP12 to transmit positive signals. It was previously recognized that Clec 5 a expression is correlated with myeloid differentiation, as Clec $5 a$ is expressed in monocytes and neutrophils, and its expression is increased by granulocytic differentiation of $32 \mathrm{Dcl} 3$ cells (21) and monocytic differentiation of U937 cells and primary progenitor cells (37). Here we present clear evidence that Clec5a supports myeloid differentiation. First, ASXL1-MT but not ASXL1-WT reduced expression of Clec5a in $32 \mathrm{Dcl} 3$ cells and impaired G-CSF-induced granulocytic differentiation. Second, in 32D3 cells expressing ASXL1-MT, myeloid differentiation was partly restored by the forced expression of Clec5a, but not by a mutant Clec5a (Clec5a-K16A) that cannot associate with DAP12. Third, knockdown of Clec5a impaired G-CSF-induced differentiation of $32 \mathrm{Dcl} 3$ cells. In addition, Clec5a expression was downregulated in the ASXL1-MT-induced MDS mice. These results indicate that downregulation of Clec $5 a$ by ASXL1-MT impairs myeloid differentiation and is involved in MDS development. Interestingly, while HOXA9 expression was high in MDS patients with ASXL1 mutations (Figure 5E), the expression level of CLEC5A was generally low in MDS patients compared with controls and did not correlate with the presence of ASXL1 mutations (Figure 3C). This suggests that CLEC5A expression is reduced in MDS by multiple causes including ASXL1 mutations, and that reduction of CLEC5A might play a general role in the pathogenesis of MDS. Prior data indicating downregulation of CLEC5A expression in acute leukemia likewise support the possibility that CLEC5A downregulation may be important in the pathogenesis of multiple subtypes of myeloid malignancies (38).

The data presented here indicate that ASXL1 mutations, which result in a truncated protein product, may (a) inhibit PRC2 function in a dominant-negative fashion and (b) promote myeloid transformation through impaired PRC2-mediated repression of posterior $H O X A$ s and miR-125a and subsequent miR-125a suppression of CLEC5A. Thus, although changes in expression of other genes caused by ASXL1 mutations may also be involved, it is documented that the derepression of HOXAs and miR-125a and the suppression of CLEC5A contribute to the development of MDS. Given the clinical importance of ASXL1 mutations, the identification of gain-of-function ASXL1 mutations is critical, as it provides a rationale for therapies aimed at targeting the expressed mutant forms of ASXL1. Finally, the data here provide further basis for the involvement of miR-125 in myeloid malignancy pathogenesis and identify an important role for CLEC5A in the pathogenesis of multiple genetic subtypes of myeloid malignancies.

\section{Methods}

Mice. C57BL/6 (Ly5.1) mice (Sankyo Labo Service Corporation) and C57BL/6 (Ly5.2) mice (Charles River Laboratories Japan) were used for BMT experiments.

Cell culture. HEK293T cells were cultured in DMEM supplemented with $10 \%$ FBS. Human leukemia cell lines were cultured in RPMI-1640 supplemented with 10\% FBS (HL60, U937, K562, KU812, TS9;22, and MEG-01 cells), RPMI-1640 supplemented with 20\% FBS (SET2, NOMO1, and MonoMac-6 cells), RPMI-1640 medium supplemented with $10 \% \mathrm{FBS} / 1 \mu \mathrm{M}$ hydrocortisone/10\% horse serum (UKE1 cells) or IMDM with $20 \%$ FBS (KBM5 cells). The murine myeloid cell lines 32Dcl3 and FDC-P1 were grown in RPMI-1640 medium supplemented with $10 \%$ FBS, antibiotics, L-glutamine, and $1 \mathrm{ng} / \mathrm{ml} \mathrm{IL-3.} \mathrm{Before} \mathrm{the} \mathrm{assays} \mathrm{for} \mathrm{proliferation} \mathrm{and}$ differentiation, the transduced 32Dcl3, HL60, or FDC-P1 cells were GFP sorted or subjected to drug selection with $1 \mu \mathrm{g} / \mathrm{ml}$ puromycin and/or $10 \mu \mathrm{g} / \mathrm{ml}$ blasticidin, if necessary.

Vector construction. We used the retrovirus vectors pMYs-FLAG-ASXL1-WT-IG and pMYs-FLAG-ASXL1-MT-IG, in which ASXL1-WT or ASXL1-MT (1934dupG;G646WfsX12 or 1900-1922del;E635RfsX15, respectively), tagged with a FLAG epitope at the $\mathrm{N}$ terminus, was inserted upstream of the IRES-EGFP cassette of PMYs-IG (39). Similarly, WT Clec5a or Clec5a-K16A was inserted upstream of the IRES-puro to generate pMYsClec5a-IP or pMYs-Clec5a-K16A-IP. ASXL1-MT was subcloned upstream of the IRES-blasticidin cassette of pMYs-IB. Likewise, Myc-tagged EZH2 was subcloned into pMYs-IG to generate pMYs-Myc-tagged EZH2-IG. To perform the mouse BMT model, we constructed pMYs-N-Ras-G12V-IG and pMYs-ASXL1-MT-IRES-nerve growth factor receptor (pMYs-ASXL1MT-IRES-NGFR). For knockdown assays, shRNA expression fragments were cloned into pMXs-U6-GFP or pMXs-U6-Puro, which were pMXs-based, self-inactivating retrovirus vectors expressing shRNA under a U6 promoter with a PGK promoter-driven GFP or puromycin-resistant gene expression. An shRNA expression cassette was constructed in the opposite direction from a GFP/Puro expression cassette. We also constructed microRNA vectors as described previously (26). Briefly, miRNA expression fragments were cloned into pMXs-EF1-Puro vector.

Transfection and retrovirus production. Retroviral production was done as described previously (40). Briefly, retroviruses were generated by transient transfection of Plat-E packaging cells with using the calcium-phosphate coprecipitation method. Cell lines such as $32 \mathrm{Dcl} 3$ were infected with the retroviruses as previously described (39).

Mutation analysis of ASXL1. Somatic mutations of ASXL1 genes were searched by sequencing exons after PCR amplification of genomic DNA, as described previously (6).

qRT-PCR. Total RNAs were treated with deoxyribonuclease I (Invitrogen) and reverse transcribed by using High Capacity cDNA Reverse Transcription Kits (Applied Biosystems) or miScript Reverse Transcription Kit (QIAGEN). qRT-PCR was performed using a Rotor-Gene Q (QIAGEN). For mRNA RT-PCR, a SYBR Premix EX Taq (Takara) was used as previously described (41). Mature miR-125a expression was measured using a miScript SYBR Green PCR kit (QIAGEN). cDNA was amplified with miR125a-specific primers (QIAGEN). Expression of RNA, U6 small nuclear 2 (RNU6B) as an internal control was used for normalization of the results. All data with error bars indicate the mean \pm SEM.

Mouse BMT. Mouse BMT was performed as described previously (40). Briefly, BM mononuclear cells were isolated from the femurs and tibias of C57BL/6 (Ly-5.1) donor mice 3 days after intraperitoneal administration of $150 \mathrm{mg} / \mathrm{kg} 5$-fluorouracil. The cells were stimulated with $50 \mathrm{ng} / \mathrm{ml}$ of mouse stem cell factor, mouse FLT3 ligand, mouse IL-6, and human thrombopoietin (all cytokines were from R\&D Systems). The prestimulated cells were infected for 60 hours with the retroviruses harboring 
pMYs-IG, pMYs-FLAG-ASXL1-MT-IG, pMYs-FLAG-ASXL1-IWT-IG, pMYsN-Ras-G12V-IG and pMYs-INGFR, or pMYs-N-Ras-G12V-IG and pMYsASXL1-MT-IRES-NGFR, using 6-well dishes coated with RetroNectin (Takara Bio). Then, $2 \times 10^{6}$ infected BM cells were injected into sublethally $\gamma$-irradiated C57BL/6 (Ly-5.2) recipient mice. Overall survival of transplanted mice was estimated using the Kaplan-Meier method. All animal studies were approved by the Animal Care Committee of the Institute of Medical Science at the University of Tokyo.

Flow cytometric analysis. Briefly, cells were stained with indicated phycoerythrin-conjugated antibodies (eBioscience). Flow cytometric analysis of the stained cells was performed with FACSCalibur Flow (BD Biosciences) equipped with FlowJo Version 7.2.4 software (TreeStar). All data with error bars indicate the mean \pm SEM.

Analysis of cell growth. Cell growth was estimated by CellTiter-Glo Luminescent Cell Viability Assay (Promega). All data with error bars indicate the mean \pm SEM.

Immunostaining. Immunostaining of $293 \mathrm{~T}$ cells transiently transfected with retrovirus constructs was performed as described previously (42). After fixation with $1.5 \%$ paraformaldehyde, cells were immunostained with rabbit anti-Flag $\mathrm{Ab}$. The cells were then stained with Alexa Fluor 546-conjugated goat anti-rabbit immunoglobulin G secondary Ab (Molecular Probes). Nuclei were counterstained with DAPI (4',6-diamidino-2phenylindole dihydrochloride). Fluorescent images were analyzed on a confocal microscope (FLUOVIEW FV300 scanning laser biological microscope JX70 system; Olympus) equipped with a SenSys/0L cold chargecoupled device camera (Olympus). An LCPlanFI ×60/1.40 NA oil was used as the objective lens. Data are representative of 3 independent experiments.

Western blot analysis. Cell lysates were subject to immunoblotting using the following antibodies: ASXL1 (clone 2049C2a; Santa Cruz Biotechnology catalog no. sc-81053; C-terminus directed), EZH2 (clone AC22; Cell Signaling Technologies catalog no. 3147), FLAG (M2 FLAG; Sigma-Aldrich catalog no. A2220), c-myc (clone 9E10; Roche catalog no. 11667203 001), H3K27me3 (CMA323) (43), total histone H3 (44), and tubulin (clone B-5-1-2; Santa Cruz Biotechnology catalog no. sc-23948). Antibodies different from the above used for immunoprecipitation include anti-EZH2 antibodies (Active Motif catalog no. 39901). Immunoprecipitation was performed in an immunoprecipitation buffer (150 mM NaCl, $50 \mathrm{mM}$ Tris pH 7.5, 1 mM EDTA, 1\% Triton, $2 \mathrm{mM}$ sodium orthovanadate, $2 \mathrm{mM}$ PMSF, $50 \mathrm{mM}$ sodium fluoride). Western blot analysis of purified histone was performed as described previously (45). Data are representative of 3 independent experiments.

ChIP assay. ChIP assays were performed as described previously (43), except for the process of cross-link and quenching reactive aldehydes. In brief, $32 \mathrm{cl} 3$ cells or sorted BM cells $\left(2-5 \times 10^{6}\right)$ were cross-linked with $1 \%$ formaldehyde for 5 minutes at room temperature and then incubated with $350 \mathrm{mM}$ glycine for 5 minutes for quenching. These assays were carried out using the following antibodies: EZH2 (Active motif catalog no. 39901), H3K4me3 (CMA304), H3K9me3 (CMA318), H3K27me3 (CMA323) (43), and ubiquityl-histone H2AK119 (clone D27C4; Cell Signaling Technologies catalog no. 8240). Quantitative PCR was performed with a Rotor-Gene Q (QIAGEN) using SYBR Premix EX Taq (Takara). All data with error bars indicate the mean \pm SEM. Data are representative of 3 independent experiments.

1. Fisher CL, et al. Additional sex combs-like 1 belongs to the enhancer of trithorax and polycomb group and genetically interacts with $\mathrm{Cbx} 2$ in mice. Dev Biol. 2010;337(1):9-15.

2. Park UH, Yoon SK, Park T, Kim EJ, Um SJ. Additional sex comb-like (ASXL) proteins 1 and 2 play opposite roles in adipogenesis via reciprocal regulation of peroxisome proliferator-activated receptor
Differentiation assay. The 32Dcl3 cells were induced to differentiate to granulocytes by removing IL-3, washing them twice in RPMI medium without IL-3, and adding G-CSF (R \& D Systems) to a final concentration of $50 \mathrm{ng} / \mathrm{ml}$. Cells were cytocentrifuged (Cytospin4; Thermo Shandon), and cell morphologies were evaluated by Giemsa staining 3 or 6 days after G-CSF addition (46). FDC-P1 cells were washed in RPMI medium and then incubated in murine GM-CSF $(10 \mathrm{ng} / \mathrm{ml})$ for 6 days (47). HL60 cells were incubated in the medium supplemented with $10^{-6} \mathrm{M}$ ATRA for 3 days. Images were obtained with a BX51 microscope and a DP12 camera (Olympus) with an Olympus UplanFl objective lens. All data with error bars indicate the mean \pm SEM. Data are representative of 3 independent experiments.

Luciferase assay. Luciferase assays to investigate whether miRNA binds to the expected target sequence were performed as described previously (26). All data with error bars indicate the mean \pm SEM.

Microarray data. Microarray data have been deposited in Gene Expression Omnibus (accession no. GSE49117 and GSE49118; http://www.ncbi.nlm.nih.gov/geo/).

Statistics. Statistical significance was calculated using the indicated tests for independent variables. All calculations were carried out using the program JMP 8.0 (SAS Institute Inc.). $P$ values of less than 0.05 were considered significant using the 2-tailed Student's $t$ test, unless otherwise noted. In box and whisker plots, the bottom and top of the box indicate the first and third quartiles, and the band inside the box is the second quartile (the median). The lowest or highest datum shows the minimum or maximum of all of data within 1.5 interquartile range of the lower or upper quartile.

Study approval. All animal studies were approved by the Animal Care Committee of the Institute of Medical Science at the University of Tokyo. MDS patients were diagnosed at Hannover Medical School. Diagnosis was based on the World Health Organization classification. Informed consent was obtained in accordance with the Declaration of Helsinki, and the studies were approved by the institutional review board of Hannover Medical School (ethical vote no. 5558) and by the ethics committees of the University of Tokyo (approval 20-10).

\section{Acknowledgments}

We thank Atsushi Iwama (Chiba University) and Makoto Nakanishi (Nagoya City University) for their helpful discussions and Shogo Yamamoto (The University of Tokyo) for microarray analysis. This work was supported by Grants-in-Aid for Scientific Research on Innovative Areas from the Ministry of Education, Culture, Sports, Science and Technology of Japan, and in part by a Grant-in-Aid for the Third-Term Comprehensive 10-Year Strategy for Cancer Control. We are grateful to Dovie Wylie for excellent language support.

Received for publication April 29, 2013, and accepted in revised form August 8, 2013.

Address correspondence to: Toshio Kitamura, Institute of Medical Science, University of Tokyo, 4-6-1 Shirokanedai, Minato-ku, Tokyo 108-8639, Japan. Phone: 81.3.5449.5759; Fax: 81.3.5449.5428; E-mail: kitamura@ims.u-tokyo.ac.jp. \{gamma\}. J Biol Chem. 2011;286(2):1354-1363.

3. Cho YS, Kim EJ, Park UH, Sin HS, Um SJ. Additional sex comb-like 1 (ASXL1), in cooperation with SRC-1, acts as a ligand-dependent coactivator for retinoic acid receptor. J Biol Chem. 2006; 281(26):17588-17598.

4. Guo S, et al. Complex oncogene dependence in microRNA-125a-induced myeloprolifera- tive neoplasms. Proc Natl Acad Sci U S A. 2012; 109(41):16636-16641.

5. Rocquain J, et al. Combined mutations of ASXL1, CBL, FLT3, IDH1, IDH2, JAK2, KRAS, NPM1, NRAS, RUNX1, TET2, and WT1 genes in myelodysplastic syndromes and acute myeloid leukemias. BMC Cancer. 2010;10:401.

6. Gelsi-Boyer V, et al. Mutations of polycomb-associ- 
ated gene ASXL1 in myelodysplastic syndromes and chronic myelomonocytic leukaemia. Br J Haematol. 2009;145(6):788-800

7. Boultwood J, et al. Frequent mutation of the polycomb-associated gene ASXL1 in the myelodysplastic syndromes and in acute myeloid leukemia. Leukemia. 2010;24(5):1062-1065.

8. Thol F, et al. Prognostic significance of ASXL1 mutations in patients with myelodysplastic syndromes. J Clin Oncol. 2011;29(18):2499-2506.

9. Gelsi-Boyer V, et al. ASXL1 mutation is associated with poor prognosis and acute transformation in chronic myelomonocytic leukaemia. BrJ Haematol. 2010;151(4):365-375

10. Abdel-Wahab O, et al. Concomitant analysis of EZH2 and ASXL1 mutations in myelofibrosis, chronic myelomonocytic leukemia and blast-phase myeloproliferative neoplasms. Leukemia. 2011; 25(7):1200-1202.

11. Abdel-Wahab O, et al. ASXL1 mutations promote myeloid transformation through loss of PRC2-mediated gene repression. Cancer Cell. 2012; 22(2):180-193.

12. Scheuermann JC, et al. Histone H2A deubiquitinase activity of the Polycomb repressive complex PR-DUB. Nature. 2010;465(7295):243-247.

13. Chung YR, Schatoff E, Abdel-Wahab O. Epigenetic alterations in hematopoietic malignancies. Int J Hematol. 2012;96(4):413-427.

14. Nikoloski G, van der Reijden BA, Jansen JH. Mutations in epigenetic regulators in myelodysplastic syndromes. Int J Hematol. 2012;95(1):8-16.

15. Vainchenker W, Delhommeau F, Constantinescu SN, Bernard OA. New mutations and pathogenesis of myeloproliferative neoplasms. Blood. 2011; 118(7):1723-1735

16. Abdel-Wahab O, Kilpivaara O, Patel J, Busque L, Levine RL. The most commonly reported variant in ASXL1 (c.1934dupG;p.Gly646TrpfsX12) is not a somatic alteration. Leukemia. 2010;24(9):1656-1657.

17. Schnittger S, et al. ASXL1 exon 12 mutations are frequent in AML with intermediate risk karyotype and are independently associated with an adverse outcome. Leukemia. 2013;27(1):82-91.

18. Schlegelberger B, Gohring G, Thol F, Heuser M. Update on cytogenetic and molecular changes in myelodysplastic syndromes. Leuk Lymphoma. 2012; 53(4):525-536.

19. Gelsi-Boyer V, Brecqueville M, Devillier R, Murati A, Mozziconacci MJ, Birnbaum D. Mutations in ASXL1 are associated with poor prognosis across the spectrum of malignant myeloid diseases. J Hematol Oncol. 2012;5:12.
20. Bakker AB, Baker E, Sutherland GR, Phillips JH, Lanier LL. Myeloid DAP12-associating lectin (MDL)- 1 is a cell surface receptor involved in the activation of myeloid cells. Proc Natl Acad Sci US A. 1999; 96(17):9792-9796.

21. Aoki N, et al. Expression and functional role of MDL-1 (CLEC5A) in mouse myeloid lineage cells. J Leukoc Biol. 2009;85(3):508-517.

22. Kim J, et al. A Myc network accounts for similarities between embryonic stem and cancer cell transcription programs. Cell. 2010;143(2):313-324.

23. MacKenzie KL, Dolnikov A, Millington M, Shounan Y, Symonds G. Mutant N-ras induces myeloproliferative disorders and apoptosis in bone marrow repopulated mice. Blood. 1999;93(6):2043-2056.

24. Gerrits A, et al. Genetic screen identifies microRNA cluster $99 \mathrm{~b} / \mathrm{let}-7 \mathrm{e} / 125 \mathrm{a}$ as a regulator of primitive hematopoietic cells. Blood. 2012;119(2):377-387.

25. Chaudhuri AA, et al. Oncomir miR-125b regulates hematopoiesis by targeting the gene Lin28A. Proc Natl Acad Sci U S A. 2012;109(11):4233-4238.

26. Enomoto Y, et al. Emu/miR-125b transgenic mice develop lethal B-cell malignancies. Lenkemia. 2011; 25(12):1849-1856.

27. Bousquet M, Harris MH, Zhou B, Lodish HF. MicroRNA miR-125b causes leukemia. Proc Natl Acad Sci U S A. 2010;107(50):21558-21563.

28. Abdel-Wahab O, Patel J, Levine RL. Clinical implications of novel mutations in epigenetic modifiers in AML. Hematol Oncol Clin North Am. 2011; 25(6):1119-1133.

29. Devillier R, et al. Acute myeloid leukemia with myelodysplasia-related changes are characterized by a specific molecular pattern with high frequency of ASXL1 mutations. Am J Hematol. 2012;87(7):659-662.

30. Dey A, et al. Loss of the tumor suppressor BAP1 causes myeloid transformation. Science. 2012; 337(6101):1541-1546.

31. Cao Q, et al. Coordinated regulation of polycomb group complexes through microRNAs in cancer. Cancer Cell. 2011;20(2):187-199.

32. Rhyasen GW, Starczynowski DT. Deregulation of microRNAs in myelodysplastic syndrome. Leukemia. 2012;26(1):13-22

33. Tassano E, Acquila M, Tavella E, Micalizzi C, Panarello C, Morerio C. MicroRNA-125b-1 and BLID upregulation resulting from a novel IGH translocation in childhood B-Cell precursor acute lymphoblastic leukemia. Genes Chromosomes Cancer. 2010;49(8):682-687.

34. Chapiro E, et al. A new recurrent translocation $\mathrm{t}(11 ; 14)$ (q24;q32) involving IGH@ and miR-125b-1 in B-cell progenitor acute lymphoblastic leukemia.
Lenkemia. 2010;24(7):1362-1364

35. Bousquet $\mathrm{M}$, et al. Myeloid cell differentiation arrest by miR-125b-1 in myelodysplastic syndrome and acute myeloid leukemia with the $\mathrm{t}(2 ; 11)(\mathrm{p} 21 ; \mathrm{q} 23)$ translocation. J Exp Med. 2008; 205(11):2499-2506

36. Ooi AG, Sahoo D, Adorno M, Wang Y, Weissman IL, Park CY. MicroRNA-125b expands hematopoietic stem cells and enriches for the lymphoid-balanced and lymphoid-biased subsets. Proc Natl Acad Sci U S A. 2010;107(50):21505-21510.

37. Gingras MC, Lapillonne H, Margolin JF. TREM-1, MDL-1, and DAP12 expression is associated with a mature stage of myeloid development. Mol Immunol. 2002;38(11):817-824.

38. Batliner J, et al. CLEC5A (MDL-1) is a novel PU.1 transcriptional target during myeloid differentiation. Mol Immunol. 2011;48(4):714-719.

39. Kitamura T, et al. Retrovirus-mediated gene transfer and expression cloning: powerful tools in functional genomics. Exp Hematol. 2003;31(11):1007-1014.

40. Watanabe-Okochi N, et al. AML1 mutations induced MDS and MDS/AML in a mouse BMT model. Blood. 2008;111(8):4297-4308.

41. Enomoto Y, et al. Characterization of leukocyte mono-immunoglobulin-like receptor 7 (LMIR7)/ CLM-3 as an activating receptor: its similarities to and differences from LMIR4/CLM-5. J Biol Chem. 2010; 285(46):35274-35283.

42. Ono R, et al. Dimerization of MLL fusion proteins and FLT3 activation synergize to induce multiple-lineage leukemogenesis. J Clin Invest. 2005; 115(4):919-929.

43. Kimura H, Hayashi-Takanaka Y, Goto Y, Takizawa $\mathrm{N}$, Nozaki N. The organization of histone H3 modifications as revealed by a panel of specific monoclonal antibodies. Cell Struct Funct. 2008; 33(1):61-73.

44. Nozawa RS, et al. Human POGZ modulates dissociation of HP1alpha from mitotic chromosome arms through Aurora B activation. Nat Cell Biol. 2010; 12(7):719-727.

45. Tanaka S, et al. Ezh2 augments leukemogenicity by reinforcing differentiation blockage in acute myeloid leukemia. Blood. 2012;120(5):1107-1117.

46. Kato N, et al. Two types of C/EBP $\alpha$ mutations play distinct but collaborative roles in leukemogenesis: lessons from clinical data and BMT models. Blood. 2011; 117(1):221-233.

47. Piazza F, Valens J, Lagasse E, Schindler C. Myeloid differentiation of FdCP1 cells is dependent on Stat5 processing. Blood. 2000; 96(4):1358-1365. 